Pacific

Journal of

Mathematics

THE RUBINSTEIN-SCHARLEMANN GRAPHIC OF A 3-MANIFOLD AS THE DISCRIMINANT SET OF A STABLE MAP

Tsuyoshi Kobayashi and Osamu Saeki

Volume 195 No. 1

September 2000 


\title{
THE RUBINSTEIN-SCHARLEMANN GRAPHIC OF A 3-MANIFOLD AS THE DISCRIMINANT SET OF A STABLE MAP
}

\author{
Tsuyoshi Kobayashi and Osamu Saeki
}

\begin{abstract}
We show that Rubinstein-Scharlemann graphics for 3-manifolds can be regarded as the images of the singular sets (: discriminant set) of stable maps from the 3-manifolds into the plane. As applications of our understanding of the graphic, we give a method for describing Heegaard surfaces in 3-manifolds by using arcs in the plane, and give an orbifold version of Rubinstein-Scharlemann's setting. Then by using this setting, we show that every genus one 1-bridge position of a nontrivial two bridge knot is obtained from a 2-bridge position in a standard manner.
\end{abstract}

\section{Introduction.}

In this paper, we show that Rubinstein-Scharlemann graphics for 3-manifolds can be regarded as the images of the singular sets (: discriminant set) of stable maps from the 3-manifolds into the plane, and as applications, we give a method for describing Heegaard surfaces in 3-manifolds by using arcs in the plane, and give an orbifold version of Rubinstein-Scharlemann's setting. Then by using this setting, we show that every genus one 1-bridge position of a non-trivial two bridge knot is obtained from a 2-bridge position in a standard manner.

In [18], Rubinstein-Scharlemann introduced a powerful machinery, which is called a graphic, for studying Heegaard splittings of 3-manifolds, and succeeded to obtain deep results on the Reidemeister-Singer distance of two strongly irreducible Heegaard splittings of a 3-manifold. We note that Rubinstein and Scharlemann derived a graphic from two Heegaard splittings of a 3-manifold via Cerf theory [5]. Then the purpose of this paper is to introduce another way for understanding the graphic. That is, we show that we can regard a graphic as the image of the singular set of a "stable map" (for definition, see Sect. 3) from the 3-manifold into the plane $\mathbb{R}^{2}$ (Theorem 4.2).

An immediate consequence of this is that we can regard a Heegaard surface as the preimage of an arc in $\mathbb{R}^{2}$, and as an application of our understanding, we first give a method for instructing a procedure for deforming one Heegaard surface to the other by using the arcs as above (Proposition 5.4), 
and describe how stabilization works in this setting (Proposition 5.6). In [19], Rubinstein and Scharlemann give a generalization of results in [18] for 3-manifolds with boundary. As the second application, we will give another formulation for generalizing the idea in $[\mathbf{1 8}]$ for link spaces. In fact, we will introduce an orbifold version of the Rubinstein-Scharlemann type argument(Sect. 6), and, by using this, we show that any genus one 1-bridge position of a 2-bridge knot is obtained from a 2-bridge position in a standard manner (Theorem 8.2).

\section{Rubinstein-Scharlemann graphic.}

Throughout this paper, we work in the differential category, and for standard terminology in 3-dimensional topology, we refer to [9], and [11].

In this section, we quickly review the setting of Rubinstein-Scharlemann's paper $[\mathbf{1 8}]$.

Let $M$ be a closed orientable 3-manifold.

Definition 2.1. We say that a decomposition $M=A \cup_{P} B$ is a (genus $g$ ) Heegaard splitting of $M$ if $A, B$ are 3-dimensional genus $g$ handlebodies in $M$ such that $M=A \cup B, A \cap B=\partial A=\partial B=P$. Then $P$ is called a (genus g) Heegaard surface of $M$.

Definition 2.2. A disk $D$ properly embedded in a handlebody $H$ is called a meridian disk of $H$ if $\partial D$ is an essential simple closed curve in $\partial H$.

Definition 2.3. A Heegaard splitting $M=A \cup_{P} B$ is stabilized, if there are meridian disks $D_{A}, D_{B}$ of $A, B$ respectively such that $\partial D_{A}$ and $\partial D_{B}$ intersects transversely in a single point.

Remark 2.4. We note that a genus $g$ Heegaard splitting $M=A \cup_{P} B$ is stabilized if and only if there exists a genus $g-1$ Heegaard splitting $A^{\prime} \cup_{P^{\prime}} B^{\prime}$ such that $A \cup_{P} B$ is obtained from $A^{\prime} \cup_{P^{\prime}} B^{\prime}$ by adding a "trivial" handle. Then we say that $M=A \cup_{P} B$ is obtained from $A^{\prime} \cup_{P^{\prime}} B^{\prime}$ by a stabilization.

Definition 2.5. A Heegaard splitting $M=A \cup_{P} B$ is reducible, if there exist meridian disks $D_{A}, D_{B}$ of $A, B$ respectively such that $\partial D_{A}=\partial D_{B}$.

Definition 2.6. A Heegaard splitting $M=A \cup_{P} B$ is weakly reducible, if there exist meridian disks $D_{A}, D_{B}$ of $A, B$ respectively such that $\partial D_{A} \cap$ $\partial D_{B}=\emptyset$.

Remark 2.7. It is easy to see that if a Heegaard splitting $M=A \cup_{P} B$ is reducible then it is weakly reducible. And it is also easy to see that if $M=A \cup_{P} B$ is stabilized and is not a genus one Heegaard splitting of the 3 -sphere $S^{3}$, then it is reducible.

Remark 2.8. It is known, by Haken [8], that if $M$ is reducible (that is, if $M$ is a connected sum of two 3 -manifolds which are not $S^{3}$ ), then any Heegaard splitting of $M$ is reducible. 
Remark 2.9. It is known, by Casson-Gordon [4], that if a Heegaard splitting $M=A \cup_{P} B$ is weakly reducible, then either it is reducible, or $M$ contains an incompressible surface.

\section{Setting of Rubinstein-Scharlemann graphic.}

Let $A \cup_{P} B, X \cup_{Q} Y$ be a pair of Heegaard splittings of a closed 3-manifold $M$. Let $\Theta_{A}, \Theta_{B}, \Theta_{X}, \Theta_{Y}$ be spines of $A, B, X, Y$ respectively such that (except for genus 0, or 1 Heegaard splittings) each vertex of $\Theta_{A}, \Theta_{B}, \Theta_{X}$, $\Theta_{Y}$ has valency 3 (see Figure 2.1). Note that for a genus 0 handlebody (:the 3-ball) $B^{3}$, we let the spine of $B^{3}$ be a point in $\operatorname{Int} B^{3}$, and for a genus 1 handlebody (:solid torus), we let the spine be a core circle of the solid torus. Then $M-\left(\Theta_{A} \cup \Theta_{B}\right)$ is homeomorphic to $P \times(0,1)$, where $P \times\{\varepsilon\}$ is close to $\Theta_{A}$, and $P \times\{1-\varepsilon\}$ is close to $\Theta_{B}$ for a small $\varepsilon>0$. We let $P_{s}$ be the surface in $M$ corresponding to $P \times\{s\}$. Then, by regarding $P_{0}=\Theta_{A}$, and $P_{1}=\Theta_{B}$, we obtain a continuous map $H: P \times I \rightarrow M$ such that $H(P, s)=P_{s}$, and we call this a sweep-out associated to $A \cup_{P} B$. Similarly we obtain a sweep-out $G: Q \times I \rightarrow M$ associated to $X \cup_{Q} Y$, and set $G(Q, t)=Q_{t}$.

Here we may suppose that $\Theta_{A} \cup \Theta_{B}$ and $\Theta_{X} \cup \Theta_{Y}, \Theta_{A} \cup \Theta_{B}$ and $G$, and $\Theta_{X} \cup \Theta_{Y}$ and $H$ are in general positions. This implies that " $H(P \times[0, \varepsilon]) \cup$ $H(P \times[1-\varepsilon, 1])$ and $G$ ", " $G(Q \times[0, \varepsilon]) \cup G(Q \times[1-\varepsilon, 1])$ and $H$ " are in a "standard" position. That is:

Regard $G(Q \times(0,1)) \stackrel{G^{-1}}{\rightarrow} Q \times(0,1) \stackrel{\text { proj. }}{\rightarrow}(0,1)$ as a height function. Then except for a neighborhood of the maxima and minima (with respect the height function) and vertices of $\Theta_{A}$, each component of $H(P \times[0, \varepsilon]) \cap Q_{t}$ is a meridian disk intersecting $\Theta_{A}$ in one point (for a small $\varepsilon>0$ ), and in the neighborhoods $Q_{t}$ looks as in Figure 2.2. The same picture holds for the other pair.

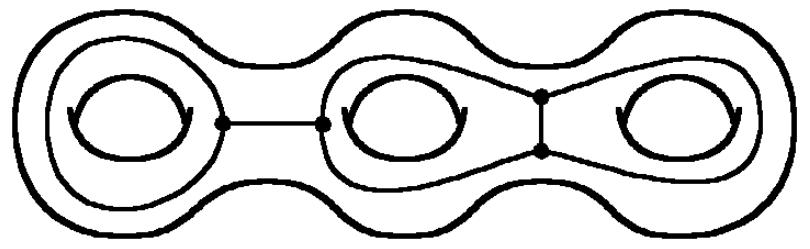

Figure 2.1. 


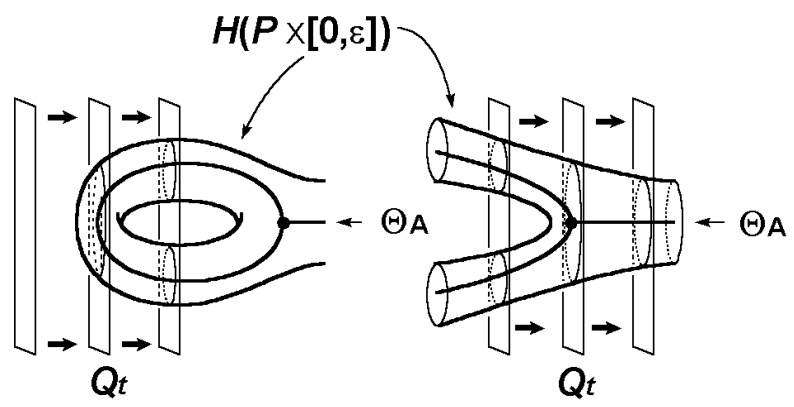

Figure 2.2.

Then, by Cerf [5], we see that for "generic" sweep-outs $H, G$ we obtain a stratification of $\operatorname{Int}(I \times I)$ which consists of four parts below.

Regions: Each region is a component of the subset of $\operatorname{Int}(I \times I)$ consisting of values $(s, t)$ such that $P_{s}$ and $Q_{t}$ intersect transversely, and this is an open set.

Edges: Each edge is a component of the subset consisting of values $(s, t)$ such that $P_{s}$ and $Q_{t}$ intersect transversely except for one nondegenerate tangent point. The tangent point is either a "center" or a "saddle". Edge is a 1-dimensional subset of $\operatorname{Int}(I \times I)$, which is monotonously increasing or decreasing.

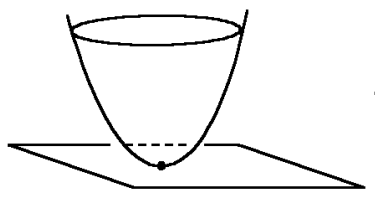

center

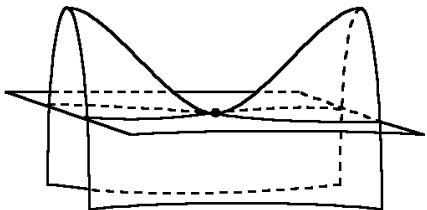

saddle

Figure 2.3.

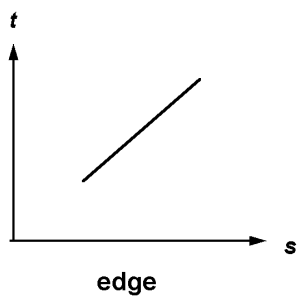

Figure 2.4.

Crossing vertices: Each crossing vertex is a component of the subset consisting of points $(s, t)$ such that $P_{s}$ and $Q_{t}$ intersect transversely except for two non-degenerate tangent points. Crossing vertex is an isolated point in $\operatorname{Int}(I \times I)$. In a neighborhood of a crossing vertex, four edges are coming in, where one can regard the crossing vertex as 
the intersection of two edges $\ell_{1}, \ell_{2}$ with the signs of the slopes of $\ell_{1}$ and $\ell_{2}$ are either different or the same.

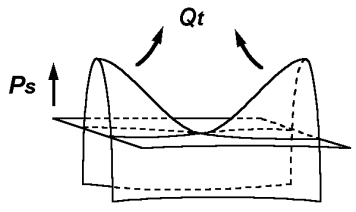

tangency corresponding to $l_{1}$

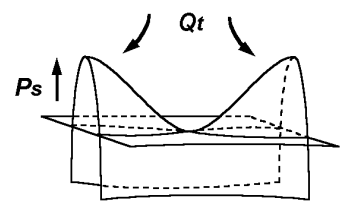

tangency corresponding to $l_{2}$

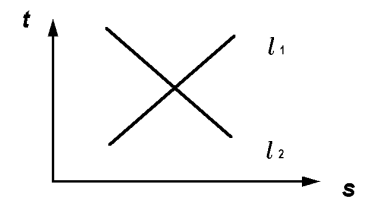

crossing vertex, different sign

Figure 2.5.

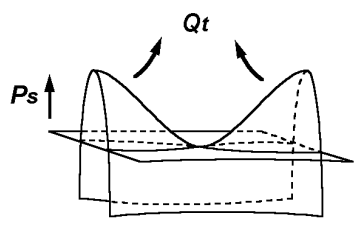

tangency corresponding to $l_{1}$

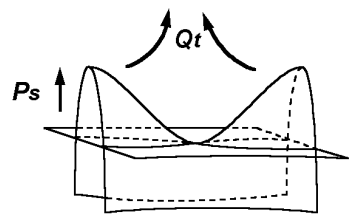

tangency corresponding to $l_{2}$

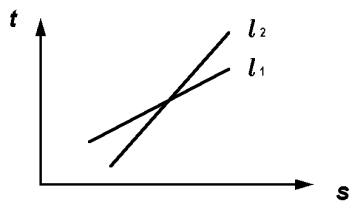

crossing vertex, same sign

Figure 2.6.

Birth-death vertices: Each birth-death vertex is a component of the subset consisting of points $(s, t)$ such that $P_{s}$ and $Q_{t}$ intersect transversely except for a single degenerate tangent point. In particular, there is a parametrization $(\lambda, \mu)$ of $I \times I$ such that $P_{s}=\{(x, y, z) \mid z=$ $0\}$, and $Q_{t}=\left\{(x, y, z) \mid z=x^{2}+\lambda+\mu y+y^{3}\right\}$. A birth-death vertex is an isolated point in $\operatorname{Int}(I \times I)$, and in a neighborhood of a birth-death vertex, two edges $\ell_{1}, \ell_{2}$ are coming in, with one from center tangency, the other from saddle tangency, and the signs of the slopes of $\ell_{1}$ and $\ell_{2}$ the same. 


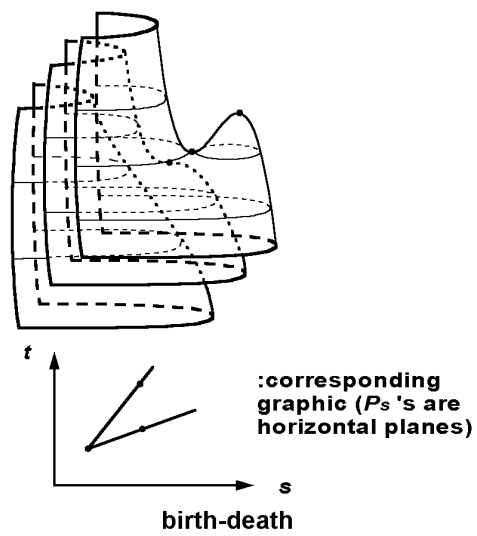

Figure 2.7.

Let $\Gamma$ be the union of edges and vertices above. By the above, $\Gamma$ is a 1-complex in $\operatorname{Int}(I \times I)$. Since we have assumed that $H, G$ are standard in a regular neighborhood of $\Theta_{A} \cup \Theta_{B} \cup \Theta_{X} \cup \Theta_{Y}$, we see that the 1-complex $\Gamma$ naturally extends to $\partial(I \times I)$. We abuse $\Gamma$ to denote this 1-complex, and we call $\Gamma$ a graphic (obtained from the sweep-outs $H, G$ ).
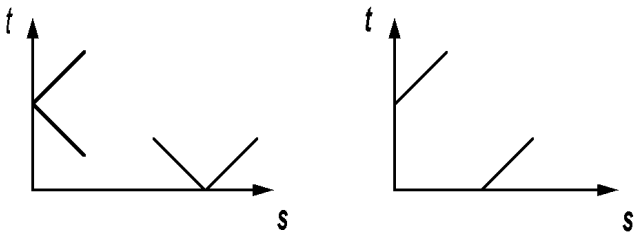

\section{$\Gamma$ near boundary}

Figure 2.8

Example 2.10. We show that there exist infinitely many 3-manifolds, and a pair of Heegaard splittings, say $A \cup_{P} B, X \cup_{Q} Y$, of each 3-manifold such that the corresponding graphic is as in Figure 2.9.

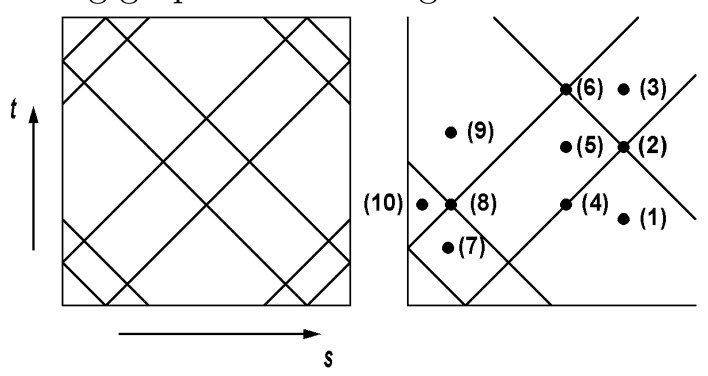

Figure 2.9.

Note that the picture admits 4 -fold $\left(\mathbb{Z}_{2} \oplus \mathbb{Z}_{2}\right)$ symmetry, and we will give an explicit description of the Heegaard surfaces belonging to the lower-left 
quarter of $I \times I$, which can be naturally extended to the whole picture under the above symmetry.

We may regard the Heegaard surface $P$ as $P_{1 / 2}$, and $Q$ as $Q_{1 / 2}$. Then $A$ and $A \cap Q_{\varepsilon}(0 \leq \varepsilon \leq 1 / 2)$ look like as follows. (Here we suppose that $A$ admits a symmetry generated by $\varphi_{1}, \varphi_{2}$ in Figure 2.10 , where $\varphi_{1}$ is an orientation preserving involution which changes the right side and the left side of $A$ with the fixed point set an arc properly embedded in $A$, and $\varphi_{2}$ is an orientation reversing involution which changes the right side and the left side of $A$ with the fixed point set a disk properly embedded in $A$.)

$A \cap Q_{0}\left(=A \cap \Theta_{X}\right)$ is a 1-complex as in Figure 2.10. Then, when $\varepsilon$ is sufficiently small, $A \cap Q_{\varepsilon}$ is the frontier of a regular neighborhood of the 1complex (the surface is homeomorphic to a torus with one hole). If we make $\varepsilon$ bigger, then we come to the point (1) of Figure 2.9 and simlutaneously four points in the boundary of a torus $Q_{\varepsilon}$ tend to four directions as in (1) of Figure 2.10.

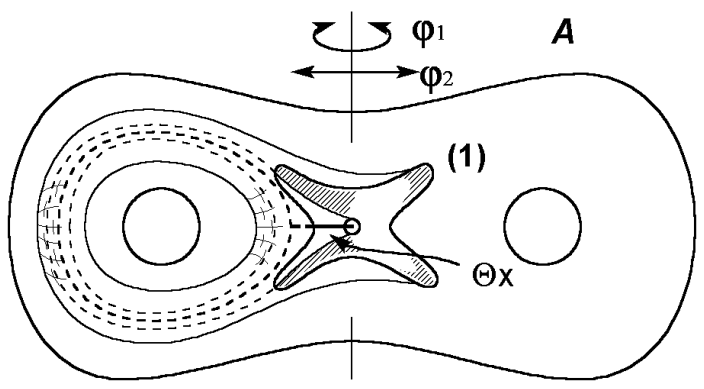

Figure 2.10.

Then, by making $\varepsilon$ bigger further, we come to the point (2) and, then, (3) $\left(: \varepsilon=1 / 2\right.$ ), where the corresponding figures of $A \cap Q_{\varepsilon}$ look as in Figure 2.11.

When we come to the point (2), the boundary component touches itself simultaneously in two places. In the right side, a band is produced, and, in the left side, the surface is boundary compressed when we pass the point (2).

Note that $A \cap Q_{1 / 2}$ is a vertical surface (which is a disk with two holes), which is located in the middle of $A$, that is, $A \cap Q_{1 / 2}$ is invariant under $\varphi_{1}$, and $\varphi_{1}$ exchanges the components of $A-Q_{1 / 2}$.

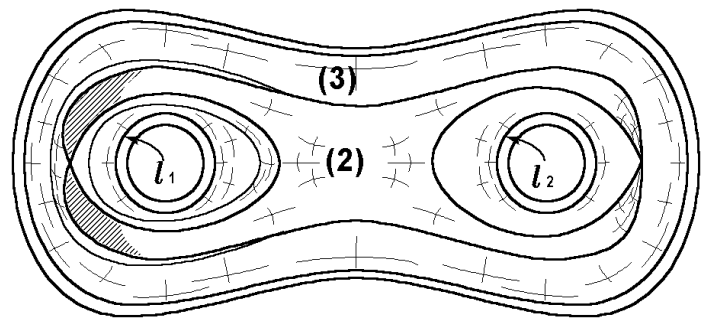

Figure 2.11. 
Figures 2.12 describes the deformations $(4) \rightarrow(5)$. When we come to (5) from (1) with passing the edge containing (4), a torus with one hole is boundary compressed and becomes an annulus.

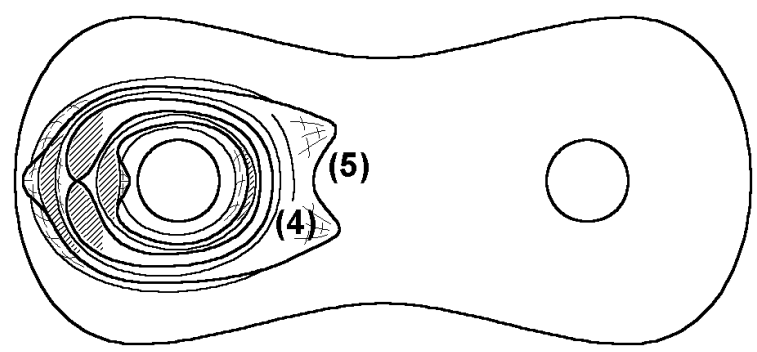

Figure 2.12.

Figure 2.13 describes the surface of (6). When we come to (6) from (5), two points of a boundary component of an annulus tend to the right side and touch and a band is produced, and simultaneously two points contained in diferent boundary components of an annulus tend to the back of $A$ and the surface is boundary copressed.

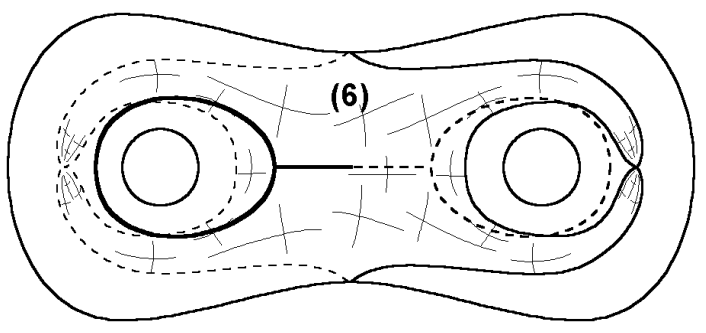

Figure 2.13.

Figures 2.14 and 2.15 describe the surfaces of (7), (8), and (9). At (7) $Q \cap A$ is an inessential disk properly embedded in $A$. When we come to (8) from (7), two points of the boundary of a disk tend to the right side and touch at a middle part of $A$, and simultaneously two points in the boundary of a disk tend to the back of $A$ and the surface is boundary comressed. As a result, an inessential disk becomes a separating essential disk in $A$.

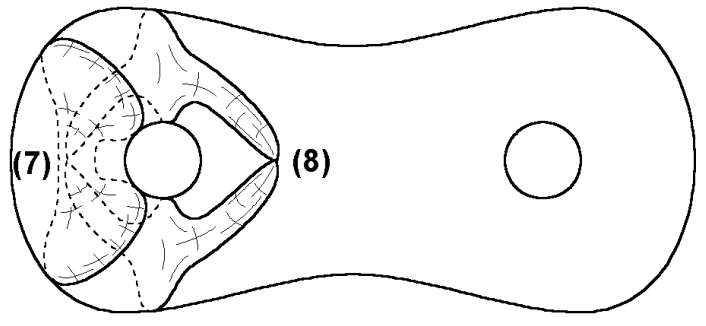

Figure 2.14. 


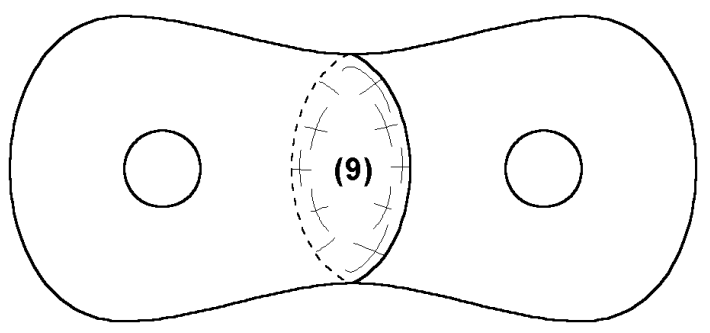

Figure 2.15.

Figure 2.16 describes (10). When we come to (10) from (7) with passing an edge, an inessential disk is boundary compressed and becomes two nonseparating disks.

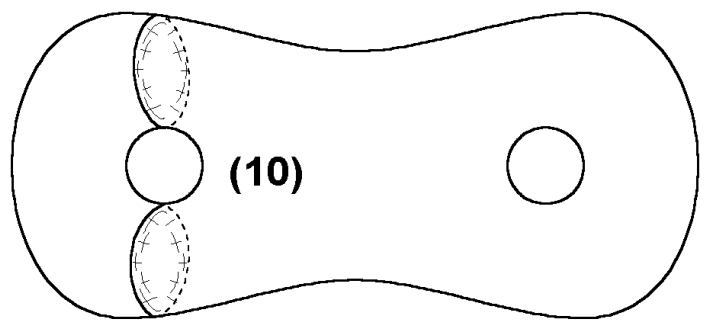

Figure 2.16.

Let $A \cap Q_{1-\varepsilon}=\varphi_{1}\left(A \cap Q_{\varepsilon}\right)(0 \leq \varepsilon \leq 1 / 2)$, which gives the whole description of $Q_{t}(0 \leq t \leq 1)$ in $A$.

Let $B$ be a copy of $A$, and $f: A \rightarrow B$ the homeomorphism induced by the identification, and $\phi=\left.f\right|_{\partial A}: \partial A \rightarrow \partial B$ the corresponding homeomorphism. Let $\mathcal{F}$ be the pattern on $\partial A$ induced by $Q_{t}$ 's. Let $M$ be the 3 -manifold obtained by attaching $A$ to $B$ by the homeomorphism $\phi \circ \varphi_{2}^{\prime}$, where $\varphi_{2}^{\prime}$ : $\partial A \rightarrow \partial A$ is isotopic to $\varphi_{2}$ with $\varphi_{2}^{\prime}(\mathcal{F})=\mathcal{F}$, and $\varphi_{2}^{\prime}\left(\ell_{2}\right)=\ell_{1}, \varphi_{2}^{\prime}\left(\ell_{1}\right)=\ell_{2}$. (Note that $M$ is actually the connected sum of two $S^{2} \times S^{1}$ 's.) We note that $\mathcal{F}$ is invariant under $\varphi_{2}$, and, hence, the surfaces $Q_{t}(0<t<1)$ in $A$ are matched to the surfaces $f \circ \varphi_{2}\left(Q_{t}\right)(0<t<1)$ in $B$ to make a system of closed surfaces, say $Q_{t}$ again, in $M$. It is directly observed from the pictures that $Q_{t}$ gives a sweep out $G: Q \times I \rightarrow M$, and, by construction, we immediately see that the corresponding graphic is as in Figure 2.9.

Then let $\ell_{1}, \ell_{2}$ be the components of $\partial\left(A \cap Q_{1 / 2}\right)$ as in Figure 2.11, and $D_{i}: \partial A \rightarrow \partial A(i=1,2)$ the Dehn twist along $\ell_{i}$. For a pair of integers $(p, q)$, we let $M_{(p, q)}$ be the manifold obtained by attaching $B$ to $A$ by the homeomorphism $\phi \circ D_{1}^{p} \circ D_{2}^{q} \circ \varphi_{2}: \partial A \rightarrow \partial B$. Since there is a regular neighborhood $N\left(\ell_{i}, \partial A\right)$ such that $\mathcal{F}$ restricted to $N\left(\ell_{i}, \partial A\right)$ is a foliation by circles parallel to $\ell_{i}$, we can arrange so that the configuration of the sweep-outs $H$ and $G$ are respected in $M_{(p, q)}$ (and, hence, the corresponding graphic is the same as above). It is easy to see that $M_{(p, q)}$ is a connected 
sum of two lens spaces $L(p, 1)$, and $L(q, 1)$, which implies the existence of infinitely many examples.

We note that it is easy to see from Figure 2.11, that the Heegaard surfaces are isotopic in each of the examples.

Example 2.11. By using the arguments in Example 2.10, we show that there exist infinitely many 3 -manifolds, and a pair of Heegaard splittings, say $A \cup_{P} B, X \cup_{Q} Y$, of each 3-manifold such that the corresponding graphic is as in Figure 2.17.
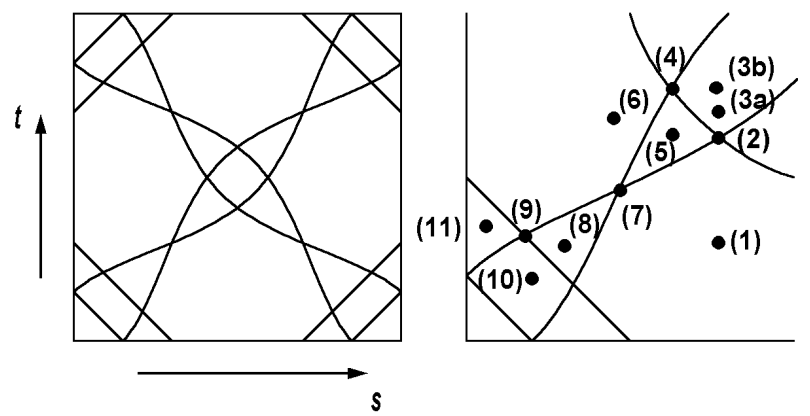

Figure 2.17.

As in Figure 2.10, $Q_{\varepsilon}$ is a torus with one hole which is the frontier of a regular neighborhood of a 1-complex for a small $\varepsilon>0$. Four points in the boundary of the torus with one hole tend to four directions according as the expansion of the regular neighborhood.

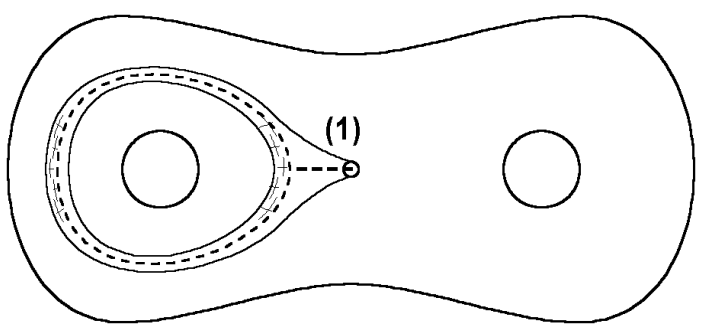

Figure 2.18.

When we come to (3a) from (1) with passing (2), a band which goes around the right handle twice is produced, and a slit in the surface which goes around the left handle twice occurs to produce a boundary compression. As a result, a torus with one hole becomes a disk with two holes. 


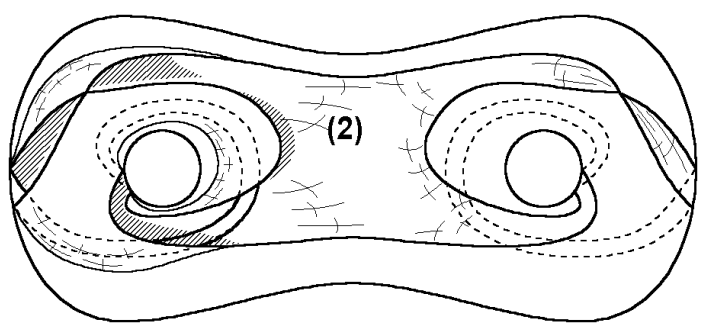

Figure 2.19.

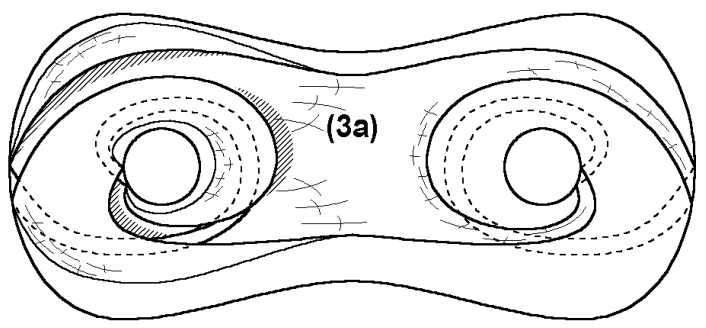

Figure 2.20a.

Note that in (3b), $Q_{1 / 2}$ is invariant with respect to the involution $\varphi_{1}$.

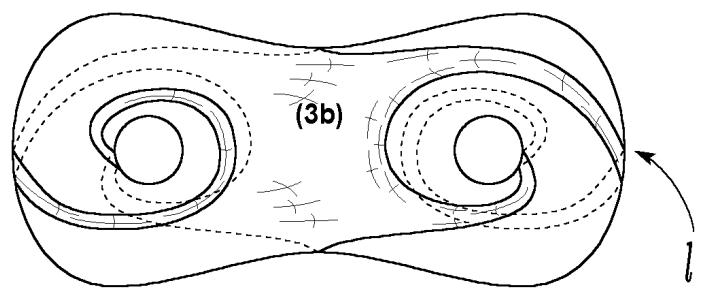

Figure 2.20b.

When we come to (6) from (3b) with passing (4), two boundary compressions occur, and a disk with two holes becomes a separating essential disk in $A$.

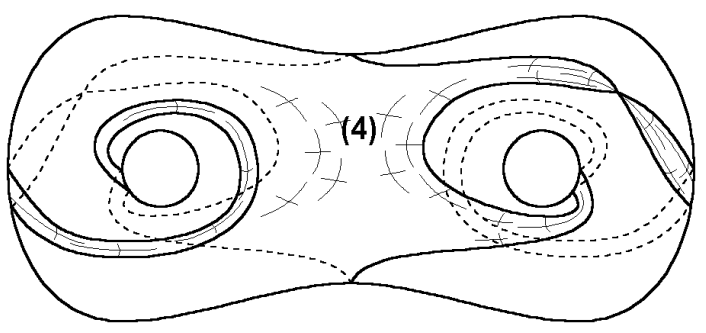

Figure 2.21. 
In the following, we show pictures with turning back to front for the convenience of drawing.

When we come to (5) from (3b) with passing an edge, the right band is boundary compressed and a disk with one hole becomes an annulus.

When we come to (6) from (5) with passing an edge, an annulus is boundary compressed to become a separating essential disk.

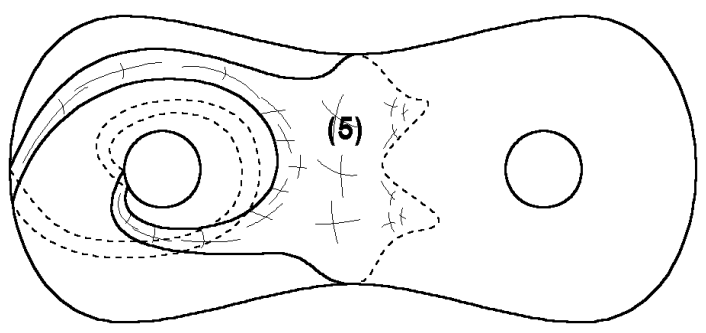

Figure 2.22.

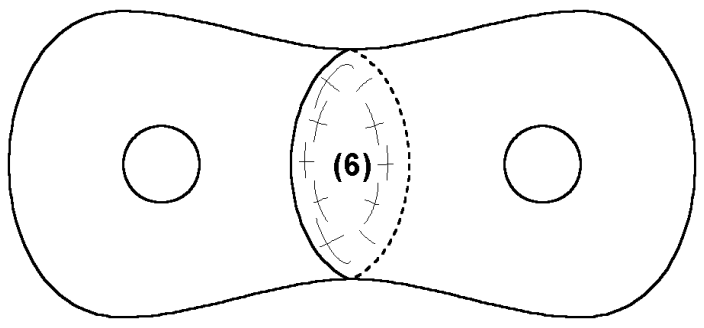

Figure 2.23.

When we come to (6) from (1) with passing (7), two bands, one of which goes around the left handle once and the other goes around the left handle twice are produced.

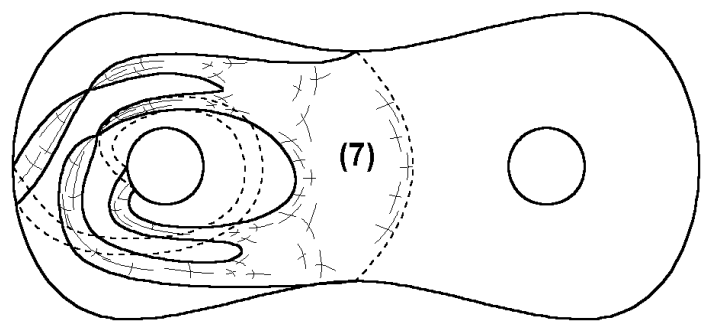

Figure 2.24.

When we come to (8) from (1) with passing an edge, a torus with one hole is boundary compressed and the punctured torus become an annulus. When we come to (8) from (6) with passing an edge, a band which goes around the left handle once is attached and the disk becomes an annulus. 


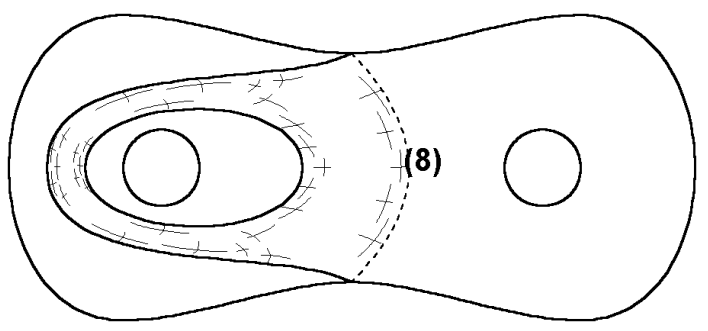

Figure 2.25.

When we come to (6) from (10) with passing (9), two points of the boundary of an inessential disk tend to the right side and touch at a middle part of $A$, and simultaneously two points in the boundary of a disk tend to the back of $A$ and touch to produce a boundary compression. As a result, an inessential disk becomes a separating essential disk in $A$.

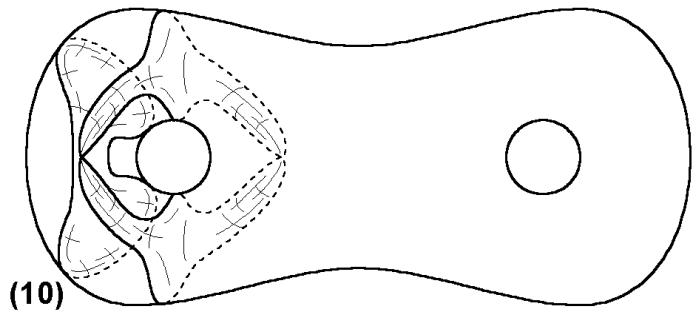

(9)

Figure 2.26.

When we come to (11) from (10) with passing an edge, an inessential disk is boundary compressed and becomes two non-separating disks.

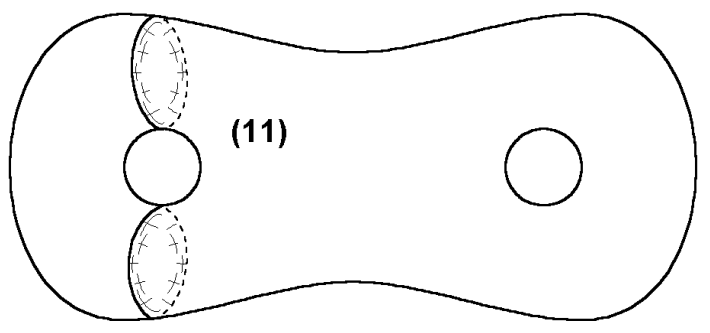

Figure 2.27.

Note that, by using the arguments in the previous example, we can show that such 3 -manifolds are obtained from $A$ and $B$ by pasting their boundaries applying the Dehn twists along $\ell$ in Figure 2.20b. Note that, as a result of this construction, we obtain 3-manifolds each of which is a union of two Seifert fibered spaces with orbit space a disk with two exceptional fibers of index two, and the exterior of a $(2,2 n)$-torus link (see [13]). Note that except in one case (case $n=0)$ they are Haken manifolds. 


\section{Stable maps.}

The purpose of this section is to show that any differentiable map from an $n$ manifold into a surface can be deformed to an "excellent" (:stable) map, and this assertion is an essential part of this paper. In the following, manifolds have countable basis and all manifolds and maps are assumed to be $C^{\infty}$.

Let $M$ be a connected $n$-dimensional manifold (possibly with boundary) with $n \geq 2$ and $N$ a surface without boundary. For a smooth map $f: M \rightarrow$ $N, S(f)$ denotes the singular set of $f$; i.e., $S(f)$ is the set of the points in $M$ where the rank of the differential $d f$ is strictly less than 2 . The discriminant set is the image of the singular set, $f(S(f))$. We denote by $C^{\infty}(M, N)$ the space of the smooth maps of $M$ into $N$ endowed with the Whitney $C^{\infty}$ topology (fine topology) (see $[\mathbf{7}, \mathbf{1 0}]$ ).

Definition 3.1. A smooth map $f: M \rightarrow N$ is stable if there exists a neighborhood $U$ of $f$ in $C^{\infty}(M, N)$ such that for each $g \in U$ there exist diffeomorphisms $H: M \rightarrow M$ and $h: N \rightarrow N$ which make the following diagram commutative.

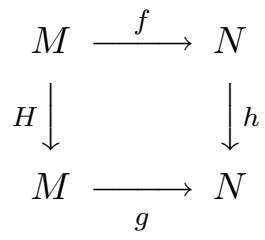

Definition 3.2. Let $f: M \rightarrow N$ be a proper smooth map of an $n$-dimensional manifold $M(n \geq 2)$ into a surface $N$ without boundary. For an open set $U$ of $N$, we say that $f$ is excellent on $U$, if $f^{-1}(U) \cap \partial M=\emptyset$ and the following conditions are satisfied: For all $p \in f^{-1}(U)$, there exist local coordinates $\left(u, x, y_{1}, \cdots, y_{n-2}\right)$ centered at $p$ and $(X, Y)$ centered at $f(p)$ such that $f$ has one of the following forms:

$$
\begin{array}{lll}
\left.L_{0}\right) \quad X \circ f=u, \quad Y \circ f=x & (p: \text { regular point }) \\
\left.L_{1}\right) \quad X \circ f=u, \quad Y \circ f= \pm x^{2}+\sum_{i=1}^{n-2} \pm y_{i}^{2} & (p: \text { fold point }) \\
\left.L_{2}\right) \quad X \circ f=u, \quad Y \circ f=u x-x^{3}+\sum_{i=1}^{n-2} \pm y_{i}^{2} & (p: \text { cusp point }) ;
\end{array}
$$

and

$\left.G_{1}\right)$ If $p \in f^{-1}(U)$ is a cusp point, then $f^{-1}(f(p)) \cap S(f)=\{p\}$,

$\left.G_{2}\right)\left.\quad f\right|_{\left(S(f) \cap f^{-1}(U)-\{\text { cusp points }\}\right)}$ is an immersion with normal crossings. 
Note that it is well known that a proper smooth map $f: M \rightarrow N$ of a manifold $M$ with $\partial M=\emptyset$ is stable if and only if $f$ is excellent on $N$ (see $[\mathbf{7}, \mathbf{1 4}])$. The terminology "excellent" comes from $[\mathbf{2 2}, \S 2]$.

The main purpose of this section is to prove the following.

Theorem 3.3. Let $f: M \rightarrow N$ be a proper smooth map. Suppose that $F$ is a closed 2-dimensional submanifold (possibly with boundary) of $N$ such that $f^{-1}(\operatorname{Int} F) \supset \partial M$. Furthermore we suppose that $f$ is excellent on a neighborhood of $\partial F$ and that $f$ is transverse to $\partial F$. Furthermore, let $\mathcal{V}$ be an arbitrary open neighborhood of $f$ in $C^{\infty}(M, N)$. Then there exists a smooth map $g: M \rightarrow N$ such that

(1) $g \in \mathcal{V}$,

(2) $g=f$ on $f^{-1}(F)=g^{-1}(F)$,

(3) $g$ is excellent on a neighborhood of $N-\operatorname{Int} F$.

Remark 3.4. In Theorem 3.3 the condition (2) is essential. In fact, if we drop the condition (2), it has already been well known.

In the following, we use the following notation. For manifolds $X$ and $Y$, $J^{k}(X, Y)$ denotes the $k$-jet bundle over $X \times Y$, i.e., $J^{k}(X, Y)=\left\{\left(x, y, j^{k}(x)\right) \mid\right.$ $x \in X, y \in Y \exists f: X \rightarrow Y: C^{\infty}$ s.t. $\left.f(x)=y\right\}$. For integers $s \geq 1$ and $k \geq 0$, $J_{s}^{k}(X, Y)$ denotes the $s$-fold $k$-jet bundle $\left(\left[\mathbf{7}, \mathrm{p}\right.\right.$. 57]). We denote by $X^{(s)}$ the subset of $X^{s}=X \times \cdots \times X$ (the $s$-fold product space of $X$ ) consisting of the elements $\left(x_{1}, \cdots, x_{s}\right)$ such that $x_{i} \neq x_{j}$ for $i \neq j$. We denote by $\pi$ : $J_{s}^{k}(X, Y) \rightarrow X^{(s)} \times Y^{s}$ the canonical projection and by $\pi_{Y}: J_{s}^{k}(X, Y) \rightarrow Y^{s}$ the natural projection to the target. We set $\Delta_{Y}^{s}=\left\{(y, \cdots, y) \in Y^{s}\right\}$ and $d: \Delta_{Y}^{s} \rightarrow Y$ is the natural identification map. Furthermore, for a smooth map $f: X \rightarrow Y, j_{s}^{k} f: X^{(s)} \rightarrow J_{s}^{k}(X, Y)$ denotes the $s$-fold $k$-jet of $f$. (For details, see [7, Chapter II, §4].)

In order to prove Theorem 3.3, we need the following.

Proposition 3.5. Let $f: X \rightarrow Y$ be a smooth map between manifolds ( $Y$ need not be a surface). Let $W$ be a submanifold of $J_{s}^{k}(X, Y)$ such that $\pi_{Y}(W) \subset \Delta_{Y}^{s}$. Suppose that $U$ is an open subset of $Y$ and that $\mathcal{V}$ is an open neighborhood of $f$ in $C^{\infty}(X, Y)$. Then there exists a smooth map $g: X \rightarrow Y$ such that

(1) $g \in \mathcal{V}$,

(2) $g=f$ on $f^{-1}(U)=g^{-1}(U)$,

(3) $j_{s}^{k} g$ is transverse to $W$ on $W \cap \pi_{Y}^{-1}\left(d^{-1}(Y-\bar{U})\right)$.

Proof. Set $W^{\prime}=W \cap \pi_{Y}^{-1}\left(d^{-1}(Y-\bar{U})\right)$, which is an open submanifold of $W$. Then there exists a countable family $\left\{W_{r}\right\}_{r=1}^{\infty}$ of open sets of $W^{\prime}$ with the following properties (a)-(f).

(a) $\cup_{r=1}^{\infty} W_{r}=W^{\prime}$. 
(b) $\overline{W_{r}} \subset W^{\prime}$, where $\overline{W_{r}}$ denotes the closure of $W_{r}$ in $J_{s}^{k}(X, Y)$.

(c) $\overline{W_{r}}$ is compact.

(d) There exist coordinate neighborhoods $U_{r, 1}, \cdots, U_{r, s}$ in $X$ and $V_{r, 1}, \ldots$, $V_{r, s}$ in $Y$ such that $\left\{\overline{U_{r, i}}\right\}_{i=1}^{s}$ are mutually disjoint and $\pi\left(\overline{W_{r}}\right) \subset U_{r, 1} \times \cdots \times$ $U_{r, s} \times V_{r, 1} \times \cdots \times V_{r, s}$.

(e) $\overline{U_{r, i}}$ is compact for $1 \leq{ }^{\forall} i \leq s$.

(f) $\overline{V_{r, i}} \cap \bar{U}=\emptyset$ for $1 \leq{ }_{i} \leq s$.

Using this family $\left\{W_{r}\right\}_{r=1}^{\infty}$ in the argument of $[7$, proof of Theorem 4.13 (p. 58)] or [15, pp. 311-312], we see that there exists a smooth map $g_{r}$ : $X \rightarrow Y$ such that $j_{s}^{k} g_{r}: X^{(s)} \rightarrow J_{s}^{k}(X, Y)$ is transverse to $W$ on $\overline{W_{r}}$ and that $g_{r}=f$ on $f^{-1}(\bar{U})=g_{r}^{-1}(\bar{U})$ in an arbitrary neighborhood of $f$ in $C^{\infty}(X, Y)$. Thus, putting

$$
\mathcal{C}_{f, U}=\left\{g \in C^{\infty}(X, Y): g=f \text { on } f^{-1}(U)=g^{-1}(U)\right\},
$$

we see that

$$
\mathcal{D}_{r}=\mathcal{C}_{f, U} \cap\left\{g \in C^{\infty}(X, Y): j_{s}^{k} g \text { is transverse to } W \text { on } \overline{W_{r}}\right\}
$$

is dense in $\mathcal{C}_{f, U}$. On the other hand, $\mathcal{D}_{r}$ is open by [7, Lemma 4.14 (p. 57)]. The proposition is proved if we show that $\cap_{r=1}^{\infty} \mathcal{D}_{r}$ is dense in $\mathcal{C}_{f, U}$. Thus we have only to show that $\mathcal{C}_{f, U}$ is a Baire space (see [7, Definition 3.2 (p. 44)]). First note that $\mathcal{C}_{f, U}$ is a closed subset of $C^{\infty}(X, Y)$. Then by imitating the proof of $\left[7\right.$, Proposition 3.3 (p. 44)], we see easily that $\mathcal{C}_{f, U}$ is a Baire space. This completes the proof.

Let $M$ and $N$ be as in Theorem 3.3. We consider some submanifolds of the (multi-)jet bundles as follows. For the jet bundle $J^{3}(M, N)$, we consider the four submanifolds $\Sigma^{n-1,0}, \Sigma^{n, 0}, \Sigma^{n-1,1,0}$ and $\Sigma^{n-1,1,1,0}$ as defined in [1] (or [7, p. 156, Sect. 5]. Note that their codimensions are equal to $n-1,2 n, n$ and $n+1$ respectively by $\left[\mathbf{1}\right.$, Theorem (6.2)]. For the multi-jet bundle $J_{2}^{3}(M, N)$, we consider

$$
\begin{aligned}
& S_{2}^{1}=\left\{\left(j^{3} f(p), j^{3} g(q)\right): f(p)=g(q), j^{3} f(p) \in \Sigma^{n-1,0}, j^{3} g(q) \in \Sigma^{n-1,0}\right\}, \\
& S_{2}^{2}=\left\{\left(j^{3} f(p), j^{3} g(q)\right): f(p)=g(q), j^{3} f(p) \in \Sigma^{n-1,0}, j^{3} g(q) \in \Sigma^{n-1,1,0}\right\}, \\
& S_{2}^{3}=\left\{\left(j^{3} f(p), j^{3} g(q)\right): f(p)=g(q), j^{3} f(p) \in \Sigma^{n-1,1,0}, j^{3} g(q) \in \Sigma^{n-1,1,0}\right\} .
\end{aligned}
$$

For the multi-jet bundle $J_{3}^{3}(M, N)$, we consider

$$
\begin{gathered}
S_{3}^{1}=\left\{\left(j^{3} f(p), j^{3} g(q), j^{3} h(r)\right): f(p)=g(q)=h(r),\right. \\
\left.j^{3} f(p), j^{3} g(q), j^{3} h(r) \in \Sigma^{n-1,0}\right\} .
\end{gathered}
$$

Note that $S_{2}^{1}, S_{2}^{2}, S_{2}^{3}$ and $S_{3}^{1}$ are easily seen to be submanifolds and that their codimensions are equal to $2 n, 2 n+1,2 n+2$ and $3 n+1$ respectively.

For a smooth map $f: M \rightarrow N$, we have the following facts:

(1) $j^{3} f$ is transverse to $\Sigma^{n-1,0}, \Sigma^{n, 0}, \Sigma^{n-1,1,0}$ and $\Sigma^{n-1,1,1,0}$ if and only if $f$ exhibits only fold and cusp points as its singularities. 
(2) Suppose $f$ satisfies (1). Then $j_{3}^{3} f$ is transverse to $S_{3}^{1}$ if and only if $\left.f\right|_{(S(f)-\{\text { cusp points }\}}$ has no multiple points of multiplicity greater than two.

(3) Suppose $f$ satisfies (1) and (2). Then $j_{2}^{3} f$ is transverse to $S_{2}^{1}$ if and only if $\left.f\right|_{(S(f)-\{\text { cusp points }\})}$ is an immersion with normal crossings (see [7, Proposition 5.6 (p. 158)]).

(4) Suppose $f$ satisfies (1). Then $j_{2}^{3} f$ is transverse to $S_{2}^{2}$ and $S_{2}^{3}$ if and only if for every cusp point $p$ of $f$, we have $f^{-1}(f(p)) \cap S(f)=\{p\}$.

Using the above facts, we obtain the following.

Lemma 3.6. Let $f: M \rightarrow N$ be a proper smooth map of an $n$-dimensional manifold $M(n \geq 2)$ into a surface $N$. For an open set $U$ of $N, f$ is excellent on $U$ if and only if $f^{-1}(U) \cap \partial M=\emptyset$ and the jets of $f$ are transverse to $\Sigma^{n-1,0}, \Sigma^{n, 0}, \Sigma^{n-1,1,0}, \Sigma^{n-1,1,1,0}, S_{2}^{1}, S_{2}^{2}, S_{2}^{3}$ and $S_{3}^{1}$ on the part corresponding to $f^{-1}(U)$.

Proof of Theorem 3.3. Set $U=\operatorname{Int} F$. By Proposition 3.5 and Lemma 3.6, we see that there exists a smooth map $g: M \rightarrow N$ such that $g \in \mathcal{V}, g=f$ on $f^{-1}(U)=g^{-1}(U)$ and that $g$ is excellent on $N-F$. Since $f$ and $g$ are continuous and $N$ is Hausdorff, we see that $g=f$ on the closure of $f^{-1}(U)$.

However, we do not know if $g$ is excellent on a neighborhood of $N-\operatorname{Int} F$. This is because there is a possibility of a point in $f^{-1}(N-F)$ being mapped into $\partial F$ by $g$. In order to exclude this possibility, we modify the argument as follows.

Since $\partial F$ is a closed submanifold of $N$, the set of maps of $M$ into $N$ transverse to $\partial F$ forms an open set of $C^{\infty}(M, N)$ (see [7, Proposition 4.5 (p. $52)]$ ). Thus we may assume that every map in the open set $\mathcal{V}$ is transverse to $\partial F$ from the beginning. Furthermore, since the set of the proper maps of $M$ into $N$ forms an open set (see [10, Theorem 1.5 (p. 38)]), we may further assume that every element of $\mathcal{V}$ is a proper map. We may further assume that each element of $\mathcal{V}$ maps $\partial M$ into $F-V$ by a similar reason, where $V$ is a closed neighborhood of $\partial F$ in $N$. Now suppose that $g \in \mathcal{V}$. Then, since $g^{-1}(\partial F)$ is a closed regular submanifold of $\operatorname{Int} M$, we see that the closure of $g^{-1}(U)$ is equal to $g^{-1}(F)$. Since $f=g$ on the closure of $f^{-1}(U)$ and $f^{-1}(U)=g^{-1}(U)$, we see that $f=g$ on $f^{-1}(F)=g^{-1}(F)$. Combining the facts that $g$ is excellent on $N-F$ and that $f$ is excellent on a neighborhood of $\partial F$, we see that $g$ is excellent on a neighborhood of $N-\operatorname{Int} F$. This completes the proof of Theorem 3.3.

Remark 3.7. Results similar to Theorem 3.3 hold for some other dimension pairs as well.

\section{Graphic as the discriminant set.}

Let $\Theta_{A}, \Theta_{B}, \Theta_{X}, \Theta_{Y}, H, G$ be as is Section 2, where $H, G$ may not be generic. In this section, we first observe that we can obtain a smooth map 
$f: M-\left(\Theta_{A} \cup \Theta_{B} \cup \Theta_{X} \cup \Theta_{Y}\right) \rightarrow I \times I$ from $H$ and $G$, and we show, by using Theorem 3.3, that $f$ can be deformed to a map $\Phi$ which is excellent in the exterior of a regular neighborhood of $\Theta_{A} \cup \Theta_{B} \cup \Theta_{X} \cup \Theta_{Y}$ by an arbitrarily small deformation. Then we see that we can obtain sweep-outs $H^{\prime}, G^{\prime}$ associated to $A \cup_{P} B, X \cup_{Q} Y$ respectively from $\Phi$, which have the feature "generic" in Sect. 2. Finally we observe that the corresponding graphic is actually the discriminant set (for the definition, see Sect. 3 ) of $\Phi$.

Let $M$ be a closed 3-manifold. In this section we consider a smooth map $f$ from $M$ to the Euclidean space $\mathbb{R}^{2}$. Recall that $S(f)$ denotes the set of singular points (or singular set) of $f$. That is,

$$
S(f)=\left\{q \in M \mid \operatorname{rank}\left(d f_{q}: T_{q} M \rightarrow T_{f(q)} \mathbb{R}^{2}\right) \leq 1\right\}
$$

Then as a special situation of Definition 3.2, we have:

Definition 4.1. Let $f: M \rightarrow \mathbb{R}^{2}$ be a smooth map. For an open set $U$ of $\mathbb{R}^{2}$, we say that $f$ is excellent on $U$ if $f^{-1}(U) \cap \partial M=\emptyset$, and the following conditions are satisfied.

(1) For each point $q \in S(f)$ there exist local coordinates $(u, x, y)$ for $q$, and $(X, Y)$ for $f(q)$ such that:

(1-1) $X \circ f=u, Y \circ f=x^{2}+y^{2}$, or

(1-2) $X \circ f=u, Y \circ f=x^{2}-y^{2}$, or

(1-3) $X \circ f=u, Y \circ f=y^{2}+u x-x^{3}$.

(2) For each cusp $q, f^{-1}(f(q)) \cap S(f)=\{q\}$ (that is, the fiber which contains $q$ does not contain another singular point).

(3) $\left.f\right|_{S(f)-\{\text { cusps }\}}$ is an immersion (possibly) with normal crossing (that is, an immersion (possibly) with transverse self intersections).

We call a singular point of type (1-1) ((1-2) resp.) a definite fold (indefinite fold resp.). Recall that a singular point of type (1-3) is called a cusp.

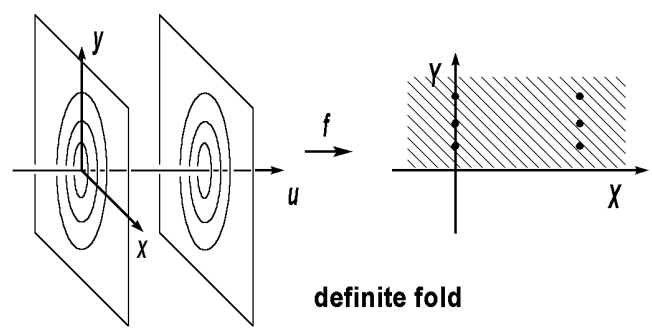

Figure 4.1. 


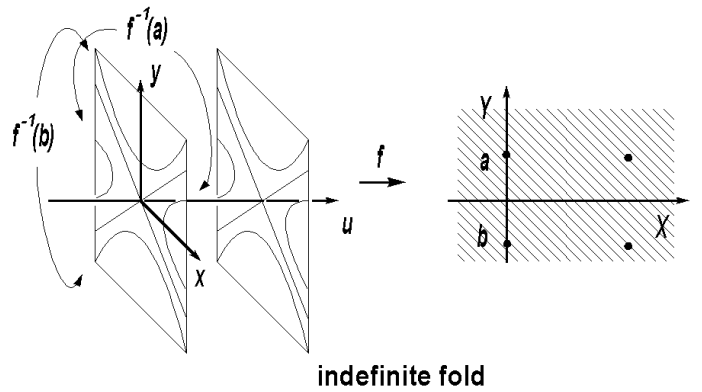

Figure 4.2.

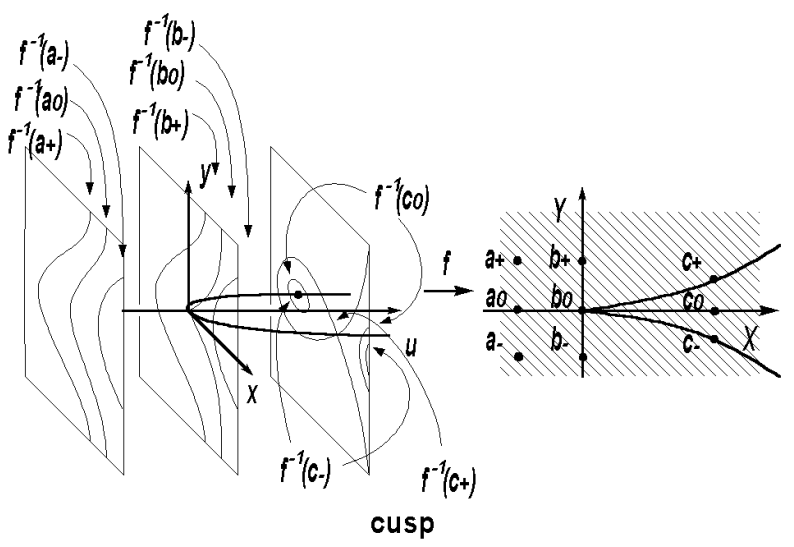

Figure 4.3.

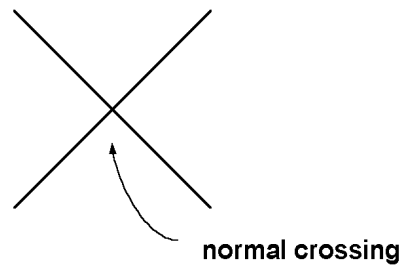

Figure 4.4.

Now we describe the relationship between graphic and excellent map. Let M, $A \cup_{P} B, X \cup_{Q} Y, \Theta_{A}, \Theta_{B}, \Theta_{X}$, and $\Theta_{Y}$ be as in Section 2. (Here we suppose that $\Theta_{A} \cup \Theta_{B}$ and $\Theta_{X} \cup \Theta_{Y}$ are in general position.)

Let $H, G$ be sweep-outs obtained from the Heegaard splittings $A \cup_{P} B$, $X \cup_{Q} Y$ respectively. We may suppose that $\left.H\right|_{P \times(0,1)}: P \times(0,1) \rightarrow M-$ $\left(\Theta_{A} \cup \Theta_{B}\right)$ and $\left.G\right|_{Q \times(0,1)}: Q \times(0,1) \rightarrow M-\left(\Theta_{X} \cup \Theta_{Y}\right)$ are smooth. Let $\Phi: M-\left(\Theta_{A} \cup \Theta_{B} \cup \Theta_{X} \cup \Theta_{Y}\right) \rightarrow I \times I$ be the map defined by:

$$
(*) \Phi(p)=(s, t) \Longleftrightarrow p \in P_{s} \cap Q_{t} .
$$

Since $\left.H\right|_{P \times(0,1)},\left.G\right|_{Q \times(0,1)}$ are smooth maps, we see that $\Phi$ is also smooth. Since $\left\{P_{s}\right\}$ and $\Theta_{X} \cup \Theta_{Y},\left\{Q_{t}\right\}$ and $\Theta_{A} \cup \Theta_{B}$ are generic, we see that: 
(*1) $H$ ( $G$ resp.) is standard (see Sect. 2) in a small regular neighborhood $N\left(\Theta_{X} \cup \Theta_{Y}\right)$ of $\Theta_{X} \cup \Theta_{Y}\left(N\left(\Theta_{A} \cup \Theta_{B}\right)\right.$ of $\Theta_{A} \cup \Theta_{B}$ resp.), and, hence, $\Phi$ is transverse to the frontier of a regular neighborhood of $\partial(I \times I)$ in $I \times I$.

By Theorem 3.3, we see that we can deform $\Phi$ in $M-\left(N\left(\Theta_{A} \cup \Theta_{B}\right) \cup\right.$ $\left.N\left(\Theta_{X} \cup \Theta_{Y}\right)\right)$ by an arbitrarily small deformation, to a map $\Phi^{\prime}$ which is excellent on the complement of the regular neighborhood of $\partial(I \times I)$ in $I \times I$, and this together with $(* 1)$ implies that $\Phi^{\prime}$ is excellent on $\operatorname{Int}(I \times I)$.

Since $\Phi^{\prime}$ is obtained from $\Phi$ by a small deformation, we may suppose:

$(* 2) p r_{1} \circ \Phi^{\prime}, p r_{2} \circ \Phi^{\prime}$ have no critical points, where $p r_{1}, p r_{2}$ : $I \times I \rightarrow I$ are the projections to the first, and second factors respectively.

By condition $(* 2)$, we see that, there exist sweep-outs $H^{\prime}, G^{\prime}$ such that $H^{\prime}(P \times\{s\})=\Phi^{\prime-1}(\{s\} \times I)$, and $G^{\prime}(Q \times\{t\})=\Phi^{\prime-1}(I \times\{t\})$. Note that $H^{\prime}, G^{\prime}$ are small deformations of $H, G$. By the definition of $H^{\prime}, G^{\prime}$, we immediately have:

$$
(*)^{\prime} \Phi^{\prime}(p)=(s, t) \Longleftrightarrow p \in H^{\prime}(P \times\{s\}) \cap G^{\prime}(Q \times\{t\}) .
$$

Then by Definition 4.1 (and the definition of the graphic in Sect. 2) we see that $H^{\prime}$ and $G^{\prime}$ are generic in the sense of Rubinstein and Scharlemann (see Sect. 2) and, by comparing Definition 4.1 and the definition of the graphic in Sect. 2, it is easy to see that the corresponding graphic is actually the image of the singular set of $\Phi^{\prime}$ on $M-\left(\Theta_{A} \cup \Theta_{B} \cup \Theta_{X} \cup \Theta_{Y}\right)$, where the image of a definite fold corresponds to a center tangency, the image of an indefinite fold corresponds to a saddle tangency, and the image of a cusp corresponds to a birth-death vertex.

Now we summarize the above results to give:

Theorem 4.2. Let $H, G$ be as above. Then, by an arbitrarily small deformation of $H$ and $G$, we obtain sweep-outs $H^{\prime}$ and $G^{\prime}$ such that:

1. The above map $\Phi^{\prime}\left(\right.$ see $\left.(*)^{\prime}\right)$ is excellent on $\operatorname{Int}(I \times I)$,

2. The maps $H^{\prime}$ and $G^{\prime}$ are generic. Hence we can obtain a graphic $\Gamma$ from $H^{\prime}$ and $G^{\prime}$, and then $\Gamma \cap \operatorname{Int}(I \times I)$ is the discriminant set of the excellent map $\left.\Phi^{\prime}\right|_{M-\left(\Theta_{A} \cup \Theta_{B} \cup \Theta_{X} \cup \Theta_{Y}\right)}$.

\section{Isotopy, and stabilization of Heegaard surfaces.}

Let $f: M \rightarrow I \times I\left(\subset \mathbb{R}^{2}\right)$ be an excellent map obtained in the previous section (which is denoted by $\Phi^{\prime}$ there). Let $\alpha: I \rightarrow \mathbb{R}^{2}$ be an embedding of the unit interval.

Definition 5.1. We say that $\alpha$ is transverse to $f$ if $\alpha$ satisfies the following two conditions: 
(1) $\alpha(\partial I) \subset \partial(I \times I)$, and $\alpha$ and $\partial(I \times I)$ are transverse (i.e., a smooth slight extension of $\alpha$ is transverse to smooth extensions of $I \times\{0\}, I \times\{1\}$, $\{0\} \times I$, and $\{0\} \times I)$,

(2) for each pair $(t, q) \in(0,1) \times M$ with $\alpha(t)=f(q)$, we have:

$$
d f_{q}\left(T_{q} M\right)+d \alpha_{t}\left(T_{t} I\right)=T_{f(q)} \mathbb{R}^{2} .
$$

Then, by Definition 4.1, it is easy to see:

Lemma 5.2. Suppose that $\alpha$ satisfies Definition 5.1 (1). Then $\alpha$ is transverse to $f$ if and only if:

For $q \in M$ with $f(q) \in \alpha(I)$, we have either one of:

1. $q$ is not a singular point of $f$,

2. $q$ is a fold point which is not a normal crossing, and $\alpha$ is transverse to the discriminant set at $f(q)$,

3. $q$ is a fold point which is mapped to a normal crossing, and $\alpha$ is transverse to the two arcs (which are local images of the singular set), or

4. $q$ is a cusp. In this case, there are two arcs in a neighborhood of $f(q)$ (which are local images of the singular set). Then there is a slight smooth extensions of the arcs, say $\ell_{1}, \ell_{2}$ such that $\alpha$ is transverse to $\ell_{1}$, and $\ell_{2}$.

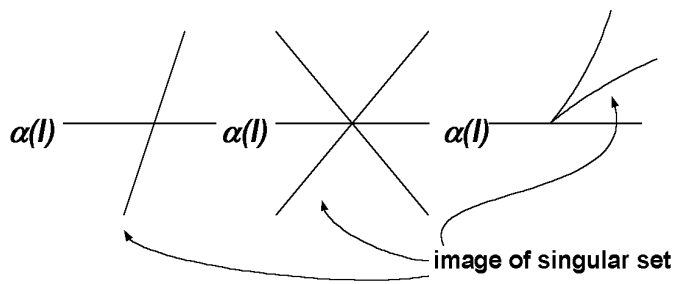

Figure 5.1.

Lemma 5.3. Suppose that $\alpha$ is transverse to $f$. Then $f^{-1}(\alpha(I))$ is a 2dimensional submanifold of $M$.

Proof. By condition Definition 5.1 (2), we see that $f^{-1}(\alpha(0,1))$ is a 2dimensional proper submanifold in $M-\left(\Theta_{A} \cup \Theta_{B} \cup \Theta_{X} \cup \Theta_{Y}\right)$. Then by condition Definition 5.1 (1), we see that $f^{-1}(\alpha[0, \varepsilon])\left(f^{-1}(\alpha[1-\varepsilon, 1])\right.$ resp.) is a disk, which cap off $f^{-1}(\alpha(0,1))$ to make a closed surface $f^{-1}(\alpha(I))$.

Proposition 5.4. Let $\alpha, \beta$ be arcs transverse to $f$. Suppose that $\alpha(I)$ is deformed to $\beta(I)$ through a sequence of moves of the following types.

(0) Ambient isotopy of $I \times I$ which fixes $\partial(I \times I) \cup \Gamma$ setwise.

(1) Passing a crossing vertex as $\alpha_{-} \rightarrow \alpha_{0} \rightarrow \alpha_{+}$in Figure 5.2. 


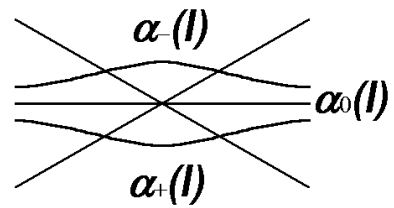

Figure 5.2.

(2) Passing a cusp as $\alpha_{-} \rightarrow \alpha_{0} \rightarrow \alpha_{+}$in Figure 5.3.

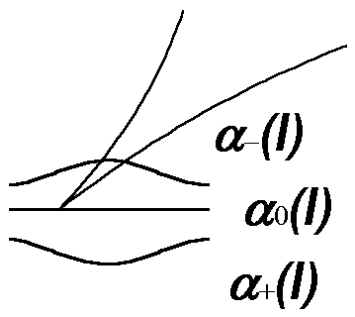

Figure 5.3.

(3) Passing a vertex in $\partial(I \times I)$ as in Figure 5.4 .
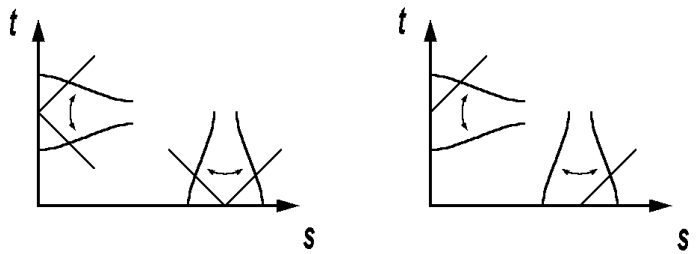

Figure 5.4.

(4) Passing a corner of $\partial(I \times I)$ as in Figure 5.5.

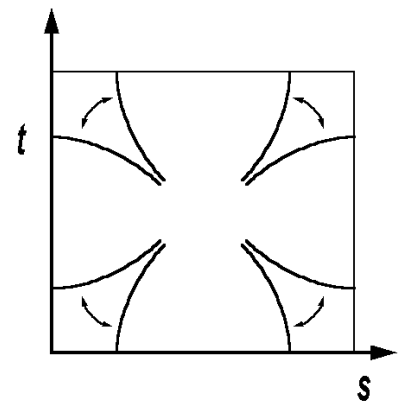

Figure 5.5.

Then the surfaces $f^{-1}(\alpha(I))$, and $f^{-1}(\beta(I))$ are isotopic in $M$.

Proof. First, we consider moves (0), (1), and (2). Suppose that $\alpha_{0}(I)$ is deformed to $\alpha_{1}(I)$ through a sequence of moves (0), (1), and (2). By Lemma 5.2, we see that there is a 1-parameter family of transverse arcs 
$\alpha_{s}(0 \leq s \leq 1)$ from $\alpha_{0}$ to $\alpha_{1}$. Then we obtain an isotopy of surfaces $f^{-1}\left(\alpha_{s}\right)$ in $M$.

Now we consider move (3). Note that the deformation (3) gives the isotopy as in Figure 5.6.
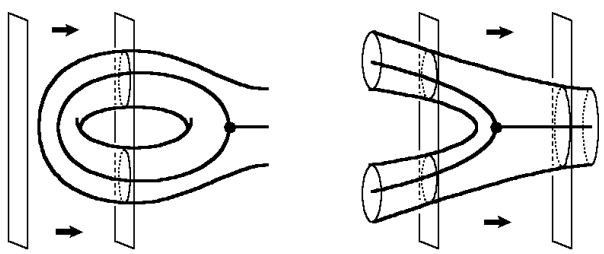

Figure 5.6.

This shows that the deformation (3) gives mutually isotopic surfaces.

Finally we consider about move (4). Note that in a neighborhood of a corner of $I \times I, P_{s}$ and $Q_{t}$ are disjoint. Hence the deformation (4) obviously gives equal surfaces.

Combining the above observations, we have the conclusion of the proposition.

As a consequence of Proposition 5.4. we have:

Corollary 5.5 (cf. Example 2.10). Suppose that the graphic obtained from $P$ and $Q$ contains a region as in Figure 5.7. Then $P$ and $Q$ are isotopic in $M$.

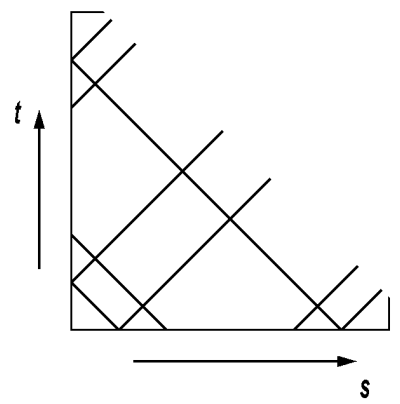

Figure 5.7.

Proof. Let $\alpha(t)=(t, \varepsilon)$, and $\beta(t)=(\varepsilon, t)$ for a small $\varepsilon>0$. It is easy to see that $\alpha(I)$ is deformed to $\beta(I)$ within the above region by applying the deformations of Proposition 5.4.

For a stabilization of a Heegaard splitting, we have:

Proposition 5.6. Let $\alpha$ be an arc transverse to $f$ such that $f^{-1}(\alpha(I))$ is a Heegaard surface. Suppose that a transverse arc $\alpha^{\prime}$ is obtained by changing 
$\alpha$ locally as in Figure 5.8. Then $f^{-1}\left(\alpha^{\prime}(I)\right)$ is a Heegaard surface which is a stabilization of $\Phi^{-1}(\alpha(I))$.

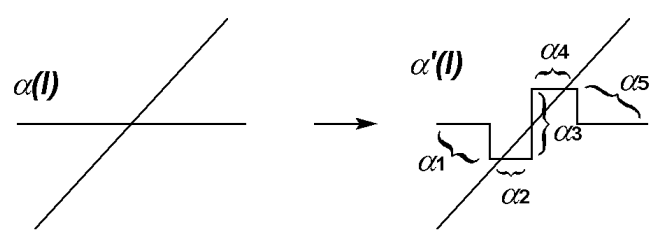

indefinite fold

Figure 5.8.

Proof. We use the following picture (Figure 5.9) for the proof. The picture corresponds to the point of the intersection of $\alpha(I)$ and the image of an indefinite fold (here $P_{s}$ 's are represented by horizontal planes).

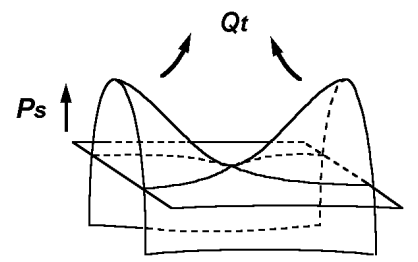

Figure 5.9.

Let $P_{i}$ be the subsurface of $\Phi^{-1}\left(\alpha^{\prime}(I)\right)$ corresponding to $\Phi^{-1}\left(\alpha_{i}\right)$, where $\alpha_{i}$ is as in Figure 5.8. It is directly observed from Figure 5.9 that each $P_{i}$ looks as in Figure 5.10.

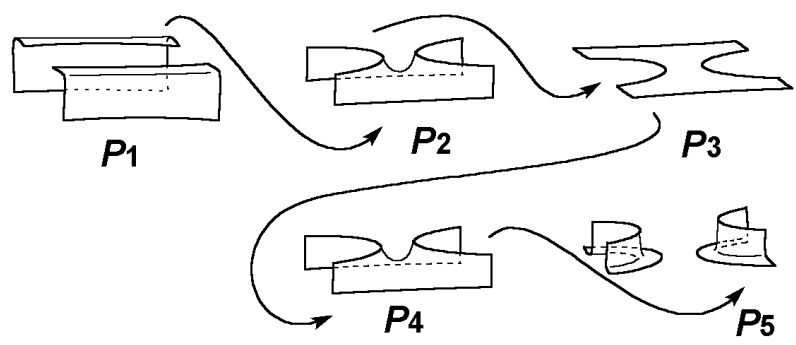

Figure 5.10.

By summing up $P_{i}$ 's, we see that $\Phi^{-1}\left(\alpha^{\prime}(I)\right)$ is a stabilization of $\Phi^{-1}(\alpha(I))$.

Corollary 5.7. Let $P$, and $Q$ be the Heegaard surfaces as in Example 2.11. Then $P$ and $Q$ become isotopic by applying one stabilization. 
Proof. We first take transverse $\operatorname{arcs} \alpha$, and $\beta$ as in Figure 5.11. Then, by Figure 5.11, we see that $\alpha(I)$ can be deformed to the arc in Figure 5.12, by one application of the deformation of Proposition 5.6, and deformations in Proposition 5.4. By reflecting the pictures in Figure 5.11 in the line connecting right-bottom corner to left-top corner, we see that $\beta(I)$ is also deformed to the arc in Figure 5.12, and this gives the conclusion.

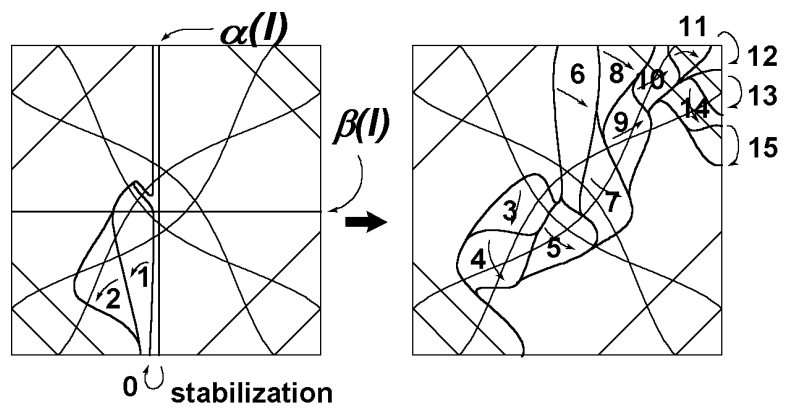

Figure 5.11.

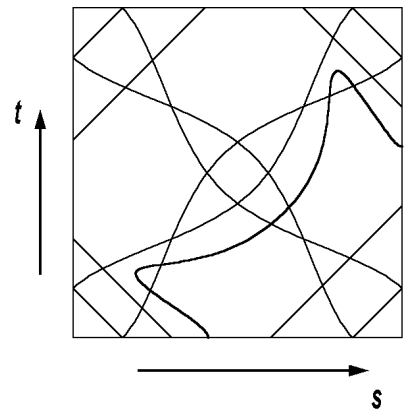

Figure 5.12.

\section{Orbifold version of Rubinstein-Scharlemann graphic.}

In this section, we formulate an orbifold version of the Rubinstein-Scharlemann setting, and show that the local labelling scheme described in [18] holds in this setting.

Let $M$ be a compact 3-manifold, $\gamma$ a union of mutually disjoint arcs or simple closed curves properly embedded in $M, F$ a surface properly embedded in $M$, which is in general position with respect to $\gamma$, and $\ell(\subset F)$ a simple closed curve with $\ell \cap \gamma=\emptyset$.

Definition 6.1. A surface $D$ is a $\gamma$-disk, if $D$ is a disk intersecting $\gamma$ in at most one transverse point.

Definition 6.2. We say that $\ell$ is $\gamma$-inessential if $\ell$ bounds a $\gamma$-disk in $F$. We say that $\ell$ is $\gamma$-essential if it is not $\gamma$-inessential. 
Let $\ell_{1}, \ell_{2}(\subset F)$ be simple closed curves with $\ell_{i} \cap \gamma=\emptyset(i=1,2)$.

Definition 6.3. We say that $\ell_{1}$ and $\ell_{2}$ are $\gamma$-parallel if $\ell_{1} \cup \ell_{2}$ bounds an annulus $A$ in $F$ such that $A \cap \gamma=\emptyset$.

Definition 6.4. We say that $D$ is a $\gamma$-compressing disk for $F$ if; $D$ is a $\gamma$-disk; and $D \cap F=\partial D$, and $\partial D$ is $\gamma$-essential in $F$. The surface $F$ is $\gamma$-compressible if it admits a $\gamma$-compressing disk, and it is $\gamma$-incompressible if it is not $\gamma$-compressible.

Let $a$ be an arc properly embedded in $F$ with $a \cap \gamma=\emptyset$.

Definition 6.5. We say that $a$ is $\gamma$-inessential if there is a subarc $b$ of $\partial F$ such that $\partial b=\partial a$, and $a \cup b$ bounds a disk $D$ in $F$ such that $D \cap \gamma=\emptyset$. We say that $a$ is weakly $\gamma$-inessential if there is a subarc $b$ of $\partial F$ such that $\partial b=\partial a$, and $a \cup b$ bounds a $\gamma$-disk $D$ in $F$.

Definition 6.6. Let $F_{1}, F_{2}$ be surfaces embedded in $M$ such that $\partial F_{1}=$ $\partial F_{2}$. We say that $F_{1}$ and $F_{2}$ are $\gamma$-parallel, if there is a submanifold $N$ in $M$ such that $\left(N, F_{1} \cap F_{2}, N \cap \gamma\right)$ is homeomorphic to $\left(F_{1} \times I, \partial F_{1} \times\{1 / 2\}, \mathcal{P} \times I\right)$ as a triple, where $\mathcal{P}$ is a union of points in $\operatorname{Int}\left(F_{1}\right)$, and $F_{1}$ ( $F_{2}$ resp.) corresponds to the closure of the component of $\partial\left(F_{1} \times I\right)-\partial F_{1} \times\{1 / 2\}$ containing $F_{1} \times\{0\}\left(F_{1} \times\{1\}\right.$ resp. $)$.

The submanifold $N$ is called a $\gamma$-parallelism between $F_{1}$ and $F_{2}$.

We say that $F$ is $\gamma$-boundary parallel if there is a subsurface $F^{\prime}$ in $\partial M$ such that $F$ and $F^{\prime}$ are $\gamma$-parallel.

Definition 6.7. Let $F_{1}, F_{2}$ be mutually disjoint surfaces in $M$ which are in general position with respect to $\gamma$. We say that $F_{1}$ and $F_{2}$ are $\gamma$-isotopic if there is an ambient isotopy $\phi_{t}(0 \leq t \leq 1)$ of $M$ such that; $\phi_{0}=i d_{M}$; $\phi_{1}\left(F_{1}\right)=F_{2}$, and; $\phi_{t}(\gamma)=\gamma$ for each $t$.

\section{Genus $g n$-bridge position.}

Let $\Gamma=\left\{\gamma_{1}, \ldots, \gamma_{n}\right\}$ be a system of mutually disjoint arcs properly embedded in $M$.

Definition 6.8. We say that $\Gamma$ is trivial if there exists a system of mutually disjoint disks $\left\{D_{1}, \ldots, D_{n}\right\}$ in $M$ such that (1) $D_{i} \cap \Gamma=\partial D_{i} \cap \gamma_{i}=\gamma_{i}$, and (2) $D_{i} \cap \partial M$ is an arc, say $\alpha_{i}$, such that $\alpha_{i}=c \ell\left(\partial D_{i}-\gamma_{i}\right)$.

Example 6.9. Let $\beta$ be a system of trivial two arcs in a 3 -ball $B$. The pair $(B, \beta)$ is often refered as 2-string trivial tangle, or a rational tangle.

Let $K$ be a link in a closed 3-manifold $M$. Let $M=A \cup_{P} B$ be a genus $g$ Heegaard splitting. Then the next definition is borrowed from [6].

Definition 6.10. We say that $K$ is in a (genus $g$ ) $n$-bridge position (with respect to the Heegaard splitting $\left.A \cup_{P} B\right)$ if $K \cap A$ ( $K \cap B$ resp.) is a system of trivial $n$ arcs in $A$ ( $B$ resp.). 
In this paper, we abbreviate genus $0 n$-bridge position to $n$-bridge position.

\section{Unknotting tunnel.}

Let $K$ be a knot in a closed 3-manifold $M$. A tunnel for $K$ is an embedded $\operatorname{arc} \sigma$ in $S^{3}$ such that $\sigma \cap K=\partial \sigma$. We say that a tunnel $\sigma$ for $K$ is unknotting if $S^{3}$-Int $N\left(K \cup \sigma, S^{3}\right)$ is a genus two handlebody.

\section{Orbifold setting.}

Let $K$ be a link in a closed 3-manifold $M$. We regard $K$ as $\gamma$ above. Let $L_{1}, L_{2}$ be a pair of mutually disjoint 1-complexes in $M$ such that:

1. Each vertex of $L_{i}$ has valency zero, one or three,

2. $\left(L_{1} \cup L_{2}\right) \cap K$ consists of the union of a (possibly, empty) sublink of $K$, and a subset of the vertices of $L_{1} \cup L_{2}$ with valency one,

3. Let $N$ be a regular neighborhood of $L_{1} \cup L_{2}$, and $E=c \ell(M-N)$. Then $(E, E \cap K)$ is homeomorphic to $(P \times(0,1), \mathcal{P} \times(0,1))$, where $P$ is a closed surface, and $\mathcal{P}$ is a finite set of (possibly empty) points in $P$.

Let $A, B$ be the closures of the components of $M-(P \times\{1 / 2\})$, where $L_{1} \subset A, L_{2} \subset B$. We say that $A \cup B$ is an orbifold Heegaard splitting of $(M, K)$. Then as in Sect. 2, we obtain a sweep-out $H: P \times I \rightarrow M$. Let $R_{1}, R_{2}$ be another pair of 1-complexes satisfying the above conditions (1), (2), and (3), and $G: Q \times I \rightarrow M$ the corresponding sweep-out. Then, as in Theorem 4.2, we may suppose that we can obtain an excellent map $f: M-\left(L_{1} \cup L_{2} \cup R_{1} \cup R_{2}\right) \rightarrow \mathbb{R}^{2}$ from $H$ and $G$ such that the graphic obtained from $H$ and $G$ is the discriminant set $f(S(f)) \subset I \times I$. Here we note that we have to slightly generalize the definition of standard position for a neighborhood of a valency one vertex (e.g. Figure 6.1), and it is easy to see the procedures in Sect. 4 work under this situation.
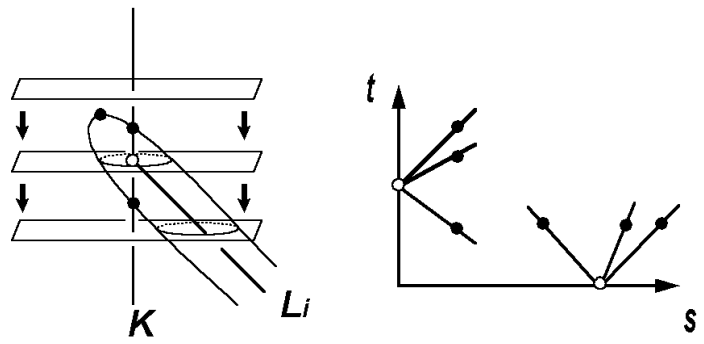

Figure 6.1.

Example 6.11. Suppose that $K$ is in a genus $g n$-bridge position with respect to a Heegaard splitting $A \cup_{P} B$. Then, by adding $n$ edges to each of the appropriate spines of $A$ and $B$, we can obtain 1-complexes satisfying the above conditions. 


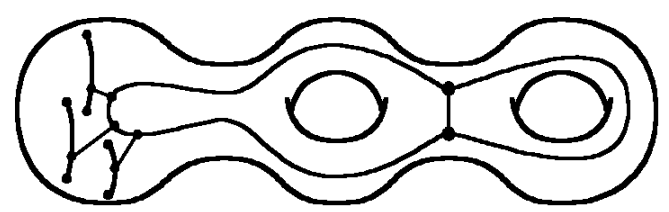

Figure 6.2.

Example 6.12. Let $K$ be a tunnel number one knot with unknotting tunnel $\tau$. Let $L_{1}=K \cup \tau$. Let $L_{2}$ be a spine of the genus two handlebody $c \ell(M-$ $N(K \cup \tau))$ with each vertex having valency three. Then $L_{1}, L_{2}$ satisfies the above conditions.

Definition 6.13. Let $H, G$ be sweep-outs as above. We say that $H$ and $G$ are $K$-comparable if $\left.f\right|_{K-\left(L_{1} \cup L_{2} \cup R_{1} \cup R_{2}\right)}: K-\left(L_{1} \cup L_{2} \cup R_{1} \cup R_{2}\right) \rightarrow \operatorname{Int}(I \times I)$ is an immersion (possibly) with normal crossing, and $f\left(K-\left(L_{1} \cup L_{2} \cup R_{1} \cup\right.\right.$ $\left.R_{2}\right)$ ) and $f(S(f))$ are in general position in $\operatorname{Int}(I \times I)$.

Proposition 6.14. By an arbitrarily small deformation on $\mathrm{K}$ rel $\left(L_{1} \cup L_{2} \cup\right.$ $R_{1} \cup R_{2}$ ) with respect to Whitney topology, we can arrange $H$ and $G$ to be K-comparable.

Proof. Note that $f^{-1}(f(S(f)))$ has the structure of a simplicial complex with dimension at most 2 (see Figure 6.3), and, hence, by an arbitrarily small deformation of $K$ with respect to Whitney topology we can arrange so that $K$ and $f^{-1}(f(S(f)))$ are in general position, that is, $K$ and the 1-skeleton are disjoint, and $K$ and $f^{-1}(f(S(f)))$ intersect transversely in a finite number of points. This shows that $f(K)$ and $f(S(f))$ intersect transversely in a finite number of points. Possibly $f(K)$ may contain a crossing vertex of the graphic $f(S(f))$. Then we further apply a small deformation to make $f(K)$ avoid crossing vertices and to make $\left.f\right|_{K-\left(L_{1} \cup L_{2} \cup R_{1} \cup R_{2}\right)}$ an immersion with normal crossing, and this gives the conclusion.
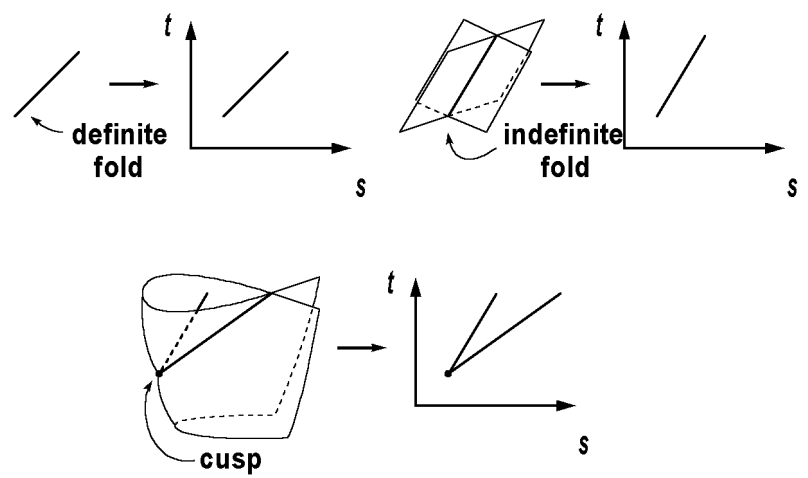

Figure 6.3. 
For a $K$-comparable pair $H$ and $G$ we can obtain a graphic $\Gamma$ as in the following manner.

Regions: A region is a component of the subset of $\operatorname{Int}(I \times I)$ consisting of values $(s, t)$ such that $P_{s}$ and $Q_{t}$ intersect transversely, and $K \cap$ $\left(P_{s} \cap Q_{t}\right)=\emptyset$.

Edges: An edge is a component of the subset consisting of values $(s, t)$ such that either:

(1) $P_{s}$ and $Q_{t}$ intersect transversely except for one non-degenerate tangent point and $K \cap\left(P_{s} \cap Q_{t}\right)=\emptyset$, or

(2) $P_{s}$ and $Q_{t}$ intersect transversely and $K \cap\left(P_{s} \cap Q_{t}\right)$ consists of one point.

By Definition 6.13, we see that edge is a 1-dimensional subset of $\operatorname{Int}(I \times I)$ which is monotonously increasing or decreasing.

Crossing vertices: A crossing vertex is a component of the subset consisting of points $(s, t)$ such that either:

(1) $P_{s}$ and $Q_{t}$ intersect transversely except for two non-degenerate points of tangency and $K \cap\left(P_{s} \cap Q_{t}\right)=\emptyset$, or

(2) $P_{s}$ and $Q_{t}$ intersect transversely except for one non-degenerate tangent point and $K \cap\left(P_{s} \cap Q_{t}\right)$ consists of one point, or

(3) $P_{s}$ and $Q_{t}$ intersect transversely and $K \cap\left(P_{s} \cap Q_{t}\right)$ consists of two points.

Note that in this setting we may (as in Sect. 2) also regard a crossing vertex to be a crossing point of two edges. This follows from the same reason as in Section 2 (Case (1)), or from the condition " $K$ and $f^{-1}(f(S(f)))$ are generic" (Case (2)), or from the condition " $\left.f\right|_{K-\left(L_{1} \cup L_{2} \cup R_{1} \cup R_{2}\right)}$ is an immersion with normal crossings" (Case (3)).

Birth-death vertices: A birth-death vertex is a component of the subset consisting of points $(s, t)$ such that $P_{s}$ and $Q_{t}$ intersect transversely except for a single degenerate tangent point and $K \cap\left(P_{s} \cap Q_{t}\right)=\emptyset$.

\section{Labelling regions of the graphic.}

Consider a region of the graphic $I \times I-\Gamma$. Then the $K$-isotopy class of $P_{s} \cap Q_{t}$ in $P_{s}$ or $Q_{t}$ is independent of the choice of $(s, t)$ in each region, and, hence, we often abbreviate $P_{s}$ by $P$, and $Q_{t}$ by $Q$.

The purpose of the rest of this section is to claim that the nature of the (local) labelling schemes discussed in [18] holds also in our setting. We assume that the reader is familiar with Sect. 4, 5 of [18].

Definition 6.15. We say that an orbifold Heegaard splitting $A \cup_{P} B$ is weakly $K$-reducible if there exist $K$-compressing disks $D_{A}, D_{B}$ for $P$ in $A$, $B$ respectively such that $\partial D_{A} \cap \partial D_{B}=\emptyset$. The orbifold Heegaard splitting $A \cup_{P} B$ is strongly $K$-irreducible if it is not weakly $K$-reducible. 
Definition 6.16. Let $(s, t)$ be a point in a region of $I \times I-\Gamma$. (Hence, $P \cap Q$ consists of a system of simple closed curves in $M$ disjoint from $K$.) Let $\mathcal{C}_{P}^{K}$ $\left(\mathcal{C}_{Q}^{K}\right.$ resp.) be the subset of the simple closed curves which are $K$-essential in $P$ ( $Q$ resp.). Then the subset $\mathcal{C}_{A}^{K}$ of $\mathcal{C}_{P}^{K}$ is defined by:

$$
\mathcal{C}_{A}^{K}=\left\{c \mid c \text { bounds a } K \text {-disk } D \text { in } Q-\mathcal{C}_{P}^{K} \text { such that } N(\partial D, D) \subset A\right\},
$$

where $N(\partial D, D)$ is a regular neighborhood of $\partial D$ in $D$.

Analogously $\mathcal{C}_{B}^{K}\left(\subset \mathcal{C}_{P}^{K}\right)$, and $\mathcal{C}_{X}^{K}, \mathcal{C}_{Y}^{K}\left(\subset \mathcal{C}_{Q}^{K}\right)$ are defined.

Lemma 6.17 (Lemma 4.3 of [18]). If $c \in \mathcal{C}_{A}^{K}$, then $c$ bounds a $K$-disk in A.

Proof. The proof is basically the same as Rubinstein-Scharlemann's except for the consideration on $K$. That is:

Let $D$ be the $K$-disk which $c$ bounds in $Q$, such that $N(\partial D, D) \subset A$. If Int $D \cap P=\emptyset$, then $D$ gives a desired $K$-disk. Suppose that $\operatorname{Int} D \cap P \neq \emptyset$. Let $\Delta(\subset D)$ be an innermost disk. Since $\operatorname{Int} D \cap \mathcal{C}_{P}^{K}=\emptyset$, we see that $\partial \Delta$ bounds a $K$-disk $\Delta^{\prime}$ in $P$. For a proof of the next claim, see Appendix A-3.

Claim. $\Delta^{\prime} \cap K=\emptyset$ if and only if $\Delta \cap K=\emptyset$. Furthermore, if $\Delta^{\prime} \cap K \neq \emptyset$, then $\Delta$ and $\Delta^{\prime}$ are $K$-parallel, i.e., $\Delta \cup \Delta^{\prime}$ bounds a 3 -ball $D^{3}$ such that $D^{3} \cap K$ is an unknotted arc.

By the claim, we see that we can apply cut and paste on $D$ using $\Delta$ and $\Delta^{\prime}$ to get a new disk $D^{\prime}$ with fewer intersections. By applying the argument finitely many times, we obtain the desired disk.

As an immediate consequence of Lemma 6.17, we have:

Corollary 6.18 (Corollary 4.4 of [18]). If there exists a region such that both $\mathcal{C}_{A}^{K}$ and $\mathcal{C}_{B}^{K}$ are non-empty, then $A \cup_{P} B$ is weakly $K$-reducible.

In the rest of this section, we suppose:

$M$ admits a 2-fold branched covering space $p: \tilde{M} \rightarrow M$ along $K$.

Lemma 6.19 (Lemma 4.5 of [18]). Suppose that $\mathcal{C}_{P}^{K}=\emptyset, \mathcal{C}_{Q}^{K}=\emptyset$, and there exists a $\partial$-reducing $K$-disk in $A$ which intersects $Q$ only in $K$-inessential simple closed curves. Suppose, moreover, that A contains a K-essential curve of $Q$. Then either $A \cup_{P} B$ is weakly $K$-reducible, or $M$ is the 3-sphere $S^{3}$ and $K$ is a trivial knot.

Proof. By Appendix A-3, we may suppose, by $K$-isotopy, that $P$ and $Q$ are disjoint, and that the $\partial$-reducing $K$-disk $D$ and $Q$ are disjoint. Without loss of generality, we may suppose that $Y$ is contained in $A$. Now consider the 2-fold branched covers (along $K) \tilde{A}, \tilde{B}, \tilde{P}, \tilde{X}, \tilde{Y}, \tilde{D}$ of $A, B, P, X, Y, D$ respectively. Note that, by the definition of an orbifold Heegaard splitting, $\tilde{A}, \tilde{B}$ are handlebodies. 
Take a maximal compression body $\tilde{C}$ of $\tilde{A}-\operatorname{Int} \tilde{Y}$ for $\partial \tilde{A}$. By the uniqueness of maximal compression body, we may suppose, by applying $\mathbb{Z}_{2}$-equivariant cut and paste arguments as in the Proof of 10.3 of [9] or [12], that $\tilde{C}$ is invariant under the covering translation $\tau$. Let $P^{\prime}$ be a component of the inner boundary of $\tilde{C}$.

If $P^{\prime}$ is a sphere, then $M$ is $S^{3}$ (see Proof of [18, Lemma 4.5]), and, by $\mathbb{Z}_{2}$-Smith Conjecture $([\mathbf{2 1}]$, or $[\mathbf{1 6}]), K$ is a trivial knot in $S^{3}$.

Suppose that $P^{\prime}$ is not a sphere. Note that $P^{\prime}$ is compressible in $\tilde{B} \cup \tilde{C}$ since $P^{\prime}$ is contained in a handlebody $\tilde{X}$ (see the proof of Lemma 4.5 of [18]). Then note that $\tilde{B} \cup_{\tilde{P}} \tilde{C}$ is a Heegaard splitting in the sense of CassonGordon [4]. Hence, by [4], there exists a compressing disk $D^{\prime}(\subset \tilde{B} \cup \tilde{C})$ for $P^{\prime}$ such that $D^{\prime} \cap \tilde{P}$ consists of a circle (hence, $D^{\prime} \cap \tilde{C}$ is an annulus).

Now we show that we can have such $D^{\prime}$ which moreover is equivariant with respect to $\tau$. We may suppose, by general position argument, that $D^{\prime}$ and $\tau\left(D^{\prime}\right)$ intersect transversely. Then, by isotopy, we may suppose that each component of $\tilde{C} \cap\left(D^{\prime} \cap \tau\left(D^{\prime}\right)\right)$ is an essential arc in the annulus $D^{\prime} \cap \tilde{C}$, (and $\tau\left(D^{\prime}\right) \cap \tilde{C}$ ). Then, by applying equivariant cut and paste arguments as in the proof of 10.3 of [9] or [12] on $D^{\prime}$, and $\tau\left(D^{\prime}\right)$, we obtain an equivariant compressing $\operatorname{disk}(\mathrm{s}) \mathcal{D}_{B}$. Since each component of $\tilde{C} \cap\left(D^{\prime} \cap \tau\left(D^{\prime}\right)\right)$ is an essential arc of $D^{\prime} \cap \tilde{C}$, we see that each component of $\mathcal{D}_{B}$ intersects $\tilde{C}$ in an annulus, (hence, intersects $\tilde{P}$ in a circle).

Then apply cut and paste arguments on $\mathcal{D}_{B} \cap \tilde{C}$ and $\tilde{D}$ to obtain a compressing disk $\tilde{D}^{\prime}(\subset \tilde{C})$ for $\tilde{P}$ such that $\tilde{D}^{\prime} \cap\left(\mathcal{D}_{B} \cap \tilde{C}\right)=\emptyset$. Then, by applying equivariant cut and paste arguments on $\tilde{D}^{\prime}$, and $\tau\left(\tilde{D}^{\prime}\right)$, we obtain equivariant $\operatorname{disk}(\mathrm{s}) \mathcal{D}_{A}(\subset \tilde{C})$ for $\tilde{P}$ such that $\mathcal{D}_{A} \cap \mathcal{D}_{B}=\emptyset$. Then $p\left(\mathcal{D}_{A}\right)$, and $p\left(\mathcal{D}_{B} \cap \tilde{B}\right)$ give weak $K$-reducibility of $K$.

\section{Labelling scheme.}

Now we mimic the procedures in $\left[\mathbf{1 8}\right.$, Section 5]. If $\mathcal{C}_{A}^{K}\left(\mathcal{C}_{B}^{K}, \mathcal{C}_{X}^{K}, \mathcal{C}_{Y}^{K}\right.$ resp.) is non-empty, then we label the region $A$ ( $B, X, Y$ resp.). If $\mathcal{C}_{P}^{K}$ and $\mathcal{C}_{Q}^{K}$ are both empty and $A$ ( $B$ resp.) contains an $K$-essential curve of $Q$, then we label the region $b$ ( $a$ resp.), and if $X$ ( $Y$ resp.) contains an essential curve of $P$, then we label the region $y$ ( $x$ resp.). By Corollary 6.18 we have:

Rule 1. If there exists a region with both labels $A$ and $B$ assigned, then $A \cup_{P} B$ is weakly $K$-reducible.

We obviously have:

Rule 2. No region can have both an upper case label and lower case label.

Next, we consider how labels change as one cross an edge of $\Gamma$.

Note that we have the following three possibilities.

1) The edge comes from center tangency. 
In this case, the regions have exactly the same label.

2) The edge comes from saddle tangency.

In this case, the effect is banding two components of $P \cap Q$, say $c_{0}$ and $c_{1}$, to make a simple closed curve, say $c$, or vice versa.

3) The edge comes from $P \cap Q \cap K$.

In this case the effect is that a component of $P \cap Q$ passes a puncture by $K$ on $P$, (and $Q)$.

Note that situation 3) did not appear in Rubinstein-Scharlemann setting. With this fact in mind, it is easy, by tracing the proof of [18, Corollary 5.1], to see:

Rule 3 ([18, Corollary 5.1]). If both labels $A$ and $B$ appear in two adjacent regions, then $A \cup_{P} B$ is weakly $K$-reducible.

Then we have:

Rule 4 ([18, Corollary 5.2]). In adjacent regions of $I \times I-\Gamma$, labels $a$ and $b$ ( $x$ and $y$ resp.) cannot appear.

Proof. Suppose that $a$ and $b$ occur opposite sides of an edge. Then arguments in the proof of [18, Corollary 5.2] show that edge does not come from saddle tangency. Then it is easy to see that this phenomena can occur only in case when $Q$ is a 2 -sphere and $Q \cap K$ consists of three points $a_{1}, a_{2}, a_{3}$ and a component of the intersection $P \cap Q$ is changed from a circle separating $a_{1}$ and $a_{2} \cup a_{3}$ to a circle separating $a_{1} \cup a_{2}$ and $a_{3}$. However this is impossible, since $Q \cap K$ must consists of even number of points.

With tracing the proof of [18, Lemma 5.3] with consideration on $K$ we easily have:

Lemma 6.20 ([18, Lemma 5.3]). Suppose, in $I \times I-\Gamma$, a region labelled $A$ is adjacent to a region labelled with a lower case letter. Then the edge represents either (1) a saddle tangency in which a band which is $K$-essential in $P$ and weakly $K$-inessential in $Q$ is attached to an intersection curve which is $K$-inessential in both $P$ and $Q$, or (2) a passing of $K$ which changes an element of $\mathcal{C}_{A}^{K}$ bounding a disk (in $P$ ) with two punctures by $K$ into a disk with one puncture by $K$.

Then we have:

Rule 5 ([18, Corollary 5.4]). Suppose, in $I \times I-\Gamma$, a region labelled $A$ is adjacent to a region labelled $b$. Then either $A \cup_{P} B$ is weakly $K$-reducible, or $M \cong S^{3}$ and $K$ is a trivial knot.

Proof. We see, by Lemma 6.20, that $A \cup_{P} B$ satisfies the assumption of Lemma 6.19, and this gives the conclusion. 
In the following, the notation A stands for, as in [18], either $a$ or $A$, and similar for $\mathrm{B}, \mathrm{X}$, and $\mathrm{Y}$. With the above rules, we see that the arguments in the proof of [18, Lemma 5.7] (it is easy to check that [18, Lemma 5.6] holds in our setting since the new phenomenon is the situation 3) in the preceding Rule 3) completely works in our setting to give:

Rule 6 ([18, Lemma 5.7]). If all letters A, B, X, and $\mathrm{Y}$ appear in quadrants of a crossing vertex of $\Gamma$, then either two opposite quadrants are unlabelled, or one of $A \cup_{P} B, X \cup_{Q} Y$ is weakly $K$-reducible, or $M \cong S^{3}$ and $K$ is a trivial knot.

By using these rules, the arguments in the proof of [18, Proposition 5.9] show (the difference here is the consideration on $K$, a possibility that three edges may be joined to a vertex in $\partial(I \times I)$ (see Figure 6.1)):

Proposition 6.21. Let $A \cup_{P} B, X \cup_{Q} Y$ be orbifold Heegaard splittings for $(M, K)$ obtained from two bridge positions as in Example 6.11. Suppose that $A \cup_{P} B, X \cup_{Q} Y$ are strongly $K$-irreducible, and $K$ is not a trivial knot in $S^{3}$. Then there is an unlabelled region in $I \times I-\Gamma$.

And, this together with Appendix A-3, and the arguments in the proof of [18, Corollary 6.2] shows:

Corollary 6.22. Let $A \cup_{P} B, X \cup_{Q} Y$ be as in Proposition 6.21. Then, by applying $K$-isotopy, we may suppose that $P$ and $Q$ intersect non-empty collection of simple closed curves which are $K$-essential in both $P$ and $Q$.

\section{2-bridge position of a 2-bridge knot.}

Let $K$ be a non-trivial 2-bridge knot (that is, $K$ is a non-trivial knot which admits a genus 0 2-bridge position). In this section, we show that the 2-bridge positions of $K$ are unique up to $K$-isotopy, which was originally proved by Schubert [20].

Theorem 7.1. Let $K$ be a non-trivial 2-bridge knot, and $P, Q$ are 2-spheres in $S^{3}$ which give 2-bridge positions of $K$. Then $P$ is $K$-isotopic to $Q$, i.e., there is an ambient isotopy $\varphi_{t}(0 \leq t \leq 1)$ of $S^{3}$ such that $(1) \varphi_{t}(K)=K$ $(0 \leq t \leq 1)$, (2) $\varphi_{0}=i d_{S^{3}}$, and $(3) \varphi_{1}(P)=Q$.

For the proof of Theorem 7.1, we prepare some lemmas, proofs of which are given in Appendix B. (For the defiition of $\beta$-essential surface, see Definition 6.2.)

Lemma 7.2 (Appendix B-1). Let $(B, \beta)$ be a 2-string trivial tangle. Let $F$ be a surface properly embedded in $B$. Suppose that $F$ is $\beta$-essential. Then $F$ is a disk which is disjoint from $\beta$, and $F$ separates the components of $\beta$. 


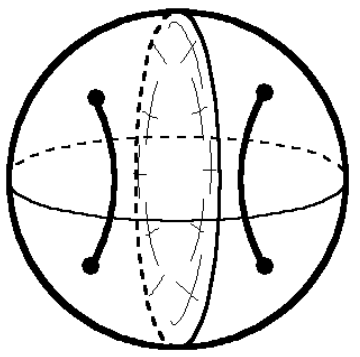

Figure 7.1.

Recall that it is often said that $(C, \gamma)$ is a rational tangle if $(C, \gamma)$ is homeomorphic to the 2 -string trivial tangle $(B, \beta)$ as a pair.

Lemma 7.3 (Appendix B-2). Let $(B, \beta)$ be a 2-string trivial tangle, and $F$ a $\beta$-incompressible surface in $B$.

Then either (0) $F$ is $\beta$-essential (see Lemma 7.2), (1) $F$ is a $\beta$-boundary parallel disk intersecting $\beta$ in at most one point, (2) $F$ is a $\beta$-boundary parallel disk intersecting $\beta$ in two points (and, hence, $F$ separates $(B, \beta)$ into the parallelism and a rational tangle), or (3) $F$ is $\beta$ boundary parallel annulus such that $F \cap \beta=\emptyset$.
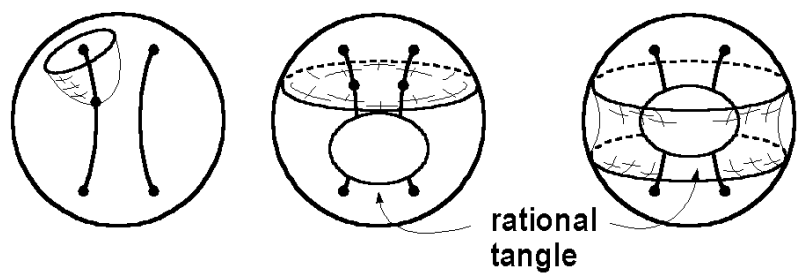

Figure 7.2.

Lemma 7.4 (Appendix B-3). Let $D$ be a $\beta$-compressible disk in $B$ such that $\partial D$ is $\beta$-essential in $\partial B$, and $D \cap \beta$ consists of two points. Then $D$ separates $(B, \beta)$ into two tangles $\left(B_{1}, \beta_{1}\right)$, and $\left(B_{2}, \beta_{2}\right)$, where $\left(B_{1}, \beta_{1}\right)$ is a rational tangle such that there is a $\beta$-essential disk $D^{\prime}$ in $\left(B_{1}, \beta_{1}\right)$ with $D \cap D^{\prime}=\emptyset$. Moreover if $\left(B_{2}, \beta_{2}\right)$ happens to be a rational tangle, then $\left(B_{2}, \beta_{2}\right)$ is a $\beta$-boundary parallelism for $D$.

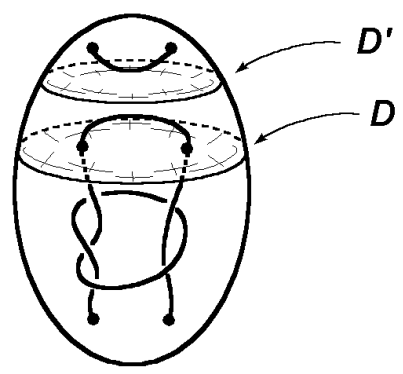

Figure 7.3. 
Let $A, B(X, Y$ resp. $)$ be the closures of the components of $S^{3}-P\left(S^{3}-Q\right.$ resp.).

Proposition 7.5. Every genus 0 Heegaard splitting of $S^{3}$ which gives a 2bridge position of $K$ is strongly $K$-irreducible.

Proof. We give the proof for $A \cup_{P} B$. Assume that $A \cup_{P} B$ is weakly $K$ reducible, and let $D_{A}, D_{B}$ be a pair of $K$-esssential disks in $A, B$ respectively such that $\partial D_{A} \cap \partial D_{B}=\emptyset$. Since $P \cap K$ consists of four points, we see that $\partial D_{A}$ and $\partial D_{B}$ are parallel in $P-K$, and this together with Lemma 7.2 implies that $K$ is a 2-component trivial link, a contradiction.

Proof of Theorem 7.1. Note that, by Proposition 7.5, $A \cup_{P} B, X \cup_{Q} Y$ are strongly $K$-irreducible. Hence, by Corollary 6.22 , we may suppose that $P$ and $Q$ intersect non-empty collection of simple closed curves which are $K$-essential in both $P$ and $Q$.

The proof is carried out by the induction on the number of the components of $P \cap Q$. The following Claims 1 and 2 give the first step of the induction.

Claim 1. If $P \cap Q$ consists of one component, then $P$ and $Q$ are $K$-isotopic. Proof. Let $D_{A}=Q \cap A, D_{B}=Q \cap B, D_{X}=P \cap X$, and $D_{Y}=P \cap Y$. Then $D_{A}, D_{B}, D_{X}, D_{Y}$ are disks each of which intersects $K$ in two points. Then we have the following cases.

Case 1. The disks $D_{A}, D_{B}, D_{X}, D_{Y}$ are $K$-incompressible in $A, B, X, Y$ respectively.

In this case, by Lemma 7.3 (2), we have:

(1) " $D_{A}$ and $D_{X}$ are $K$-parallel in $A$ " or " $D_{A}$ and $D_{Y}$ are $K$-parallel in $A$ ", (2) " $D_{B}$ and $D_{X}$ are $K$-parallel in $B$ " or " $D_{B}$ and $D_{Y}$ are $K$-parallel in $B$ ", (3) " $D_{X}$ and $D_{A}$ are $K$-parallel in $X$ " or " $D_{X}$ and $D_{B}$ are $K$-parallel in $X "$, and

(4) " $D_{Y}$ and $D_{A}$ are $K$-parallel in $Y$ " or " $D_{Y}$ and $D_{B}$ are $K$-parallel in $Y$ ". It is easy to see that the above 4 conditions imply either one of:

(1) " $D_{A}$ and $D_{X}$ are $K$-parallel in $A$ (and, $X$ ), and $D_{B}$ and $D_{Y}$ are $K$ parallel in $B$ (and, $Y$ )", or

(2) " $D_{A}$ and $D_{Y}$ are $K$-parallel in $A$ (and, $Y$ ), and $D_{B}$ and $D_{X}$ are $K$ parallel in $B$ (and, $X$ )".

Since the argument is symmetric, we may suppose that (1) holds. Then, by using the parallelisms, we can move $D_{A}$ to $D_{X}$, and $D_{B}$ to $D_{Y}$ to give a desired $K$-isotopy.

Case 2. One of the disks $D_{A}, D_{B}, D_{X}$, or $D_{Y}$ is $K$-compressible in $A, B$, $X$, or $Y$. 
Without loss of generality, we may suppose that $D_{Y}$ is $K$-compressible in $Y$, and the $K$-compressing disk is contained in $B$. Note that this implies that $D_{B}$ is $K$-compressible in $B$. Note moreover that $D_{X}$ is $K$-incompressible in $X$, since $D_{X}$ separates the boundary components of each component of $K \cap X$ in $X$, and similarly $D_{A}$ is $K$-incompressible in $A$. Then, by Lemma 7.3 (2) and the last half of Lemma 7.4, we see that $D_{B}$ and $D_{X}$ are $K$-parallel in $B$ (and, $X$ ). Similarly we can show that $D_{A}$ and $D_{Y}$ are $K$-parallel in $A$ (and, $Y$ ). Hence we can obtain a desired $K$-isotopy as in Case 1.

This completes the proof of Claim 1.

Claim 2. If $P \cap Q$ consists of two components, then $P$ and $Q$ are $K$-isotopic.

Proof. Let $D_{1}, A_{0}, D_{2}$ be the closures of the components of $P-(P \cap Q)$ such that $D_{1}$ and $D_{2}$ are disks, and $A_{0}$ is an annulus. Without loss of generality, we may suppose that $D_{1} \cup D_{2}$ is contained in $X$, and $A_{0}$ is contained in $Y$.

Subclaim. Either $D_{1}$ or $D_{2}$ is $K$-boundary parallel in $X$.

Proof. If $D_{1}$ or $D_{2}$ is $K$-incompressible, then this immediately follows from Lemma 7.3 (2). Suppose that $D_{1}$ and $D_{2}$ are $K$-compressible. Let $B_{1}^{3}$ be the closure of the component of $X-D_{1}$ which corresponds to $B_{2}$ in Lemma 7.4. By exchanging suffix, if necessary, we may suppose that $\operatorname{Int}\left(B_{1}^{3}\right) \cap P=$ $\emptyset$. Without loss of generality, we may suppose that $B_{1}^{3}$ is contained in $A$. Let $D^{1}=B_{1}^{3} \cap Q$. Since $D^{1}$ separates the boundary components of each component of $K \cap A$ in $A$, we see that $D^{1}$ is $K$-essential in $A$. Hence, by Lemma 7.3 (2) and the last half of Lemma 7.4, we see that $B_{1}^{3}$ is a $K$-parallelism.

Let $B_{1}^{3}$ be the parallelism between $D_{1}$ and $\partial X$ obtained in Subclaim. Then we can push $D_{1}$ out of $X$ along the parallelism, and we have the conclusion by Claim 1 .

This completes the proof of Claim 2.

Completion of Proof. Suppose that $\sharp(P \cap Q) \geq 3$. Note that the components of $P \cap Q$ are mutually $K$-parallel in $P$. Let $D_{1}, A_{1}, \ldots, A_{m}, D_{2}$ be the closures of the components of $P-(P \cap Q)$ such that $D_{1}, D_{2}$ are disks and $A_{1}, \ldots, A_{m}$ are annuli that are located on $P$ in this order. Suppose that $D_{1}$ or $D_{2}$ is $K$-boundary parallel in $X$ or $Y$. Then, by using the arguments in the proof of Claim 2, we can reduce $\sharp(P \cap Q)$, to give the conclusion. Suppose that $D_{1}$ and $D_{2}$ are not $K$-boundary parallel in $X$ and $Y$. By Lemma $7.3(2)$, this implies that $D_{1}$ and $D_{2}$ are $K$-compressible.

Claim 3. Both $D_{1}$ and $D_{2}$ are contained in the closure of a component of $S^{3}-Q$, say $X$. And each component of $P \cap Y$ is a $K$-incompressible annulus. 
Proof. Assume that $D_{1}$ is contained in $X$, and $D_{2}$ is contained in $Y$. By applying $K$-compression on $D_{1}\left(D_{2}\right.$ resp.) we obtain a $K$-essential disk $E_{1}$ ( $E_{2}$ resp.) in $X$ ( $Y$ resp.) such that $\partial E_{i}=\partial D_{i}$. Note that $E_{1}$ ( $E_{2}$ resp.) separates the components of $K \cap X$ ( $K \cap Y$ resp.). This implies that $K$ is a 2-component trivial link, a contradiction. Hence we may suppose that $D_{1}$ and $D_{2}$ are contained in $X$. Let $A_{i}$ be a component of $P \cap Y$. Assume that $A_{i}$ is $K$-compressible in $Y$. Then, by applying $K$-compression on $A_{i}$, we obtain two $K$-essential disks in $Y$. Then, by the above argument, we see that $K$ is a 2 -component trivial link, a contradiction.

Claim 3 together with Lemma 7.3 (3) implies that each component of $P \cap Y$ is a $K$-boundary parallel annulus in $Y$. Take an outermost one of $P \cap Y$, say $A_{j}$, and push $A_{j}$ out of $Y$ along the parallelism. This reduces $\sharp(P \cap Q)$ by two, and we have the conclusion by the assumption of the induction.

This completes the proof of Theorem 7.1.

\section{Genus one 1-bridge position of a 2-bridge knot.}

For a 2-bridge knot $K$ we can obtain four genus one 1-bridge positions of $K$ as follows.

Let $A \cup_{P} B$ be the Heegaard splitting which gives the 2-bridge position. Then let $a_{1}, a_{2}, b_{1}, b_{2}$ be the closures of the components of $K-P$, where $a_{1} \cup a_{2}\left(b_{1} \cup b_{2}\right.$ resp.) is contained in $A$ ( $B$ resp.). Let $T_{1}=A \cup N\left(b_{1}, B\right)$, $\alpha_{1}=a_{1} \cup b_{1} \cup a_{2}, T_{2}=c \ell\left(B-N\left(b_{1}, B\right)\right)$, and $\alpha_{2}=b_{2}$. Then each $T_{i}$ is a solid torus and $\alpha_{i}$ is a trivial arc in $T_{i}$, and, hence, $T_{1} \cup T_{2}$ gives genus one 1 -bridge position of $K$. Moreover, by using $a_{1}, a_{2}, b_{2}$ for $b_{1}$, we can obtain other three genus one 1-bridge positions of $K$.

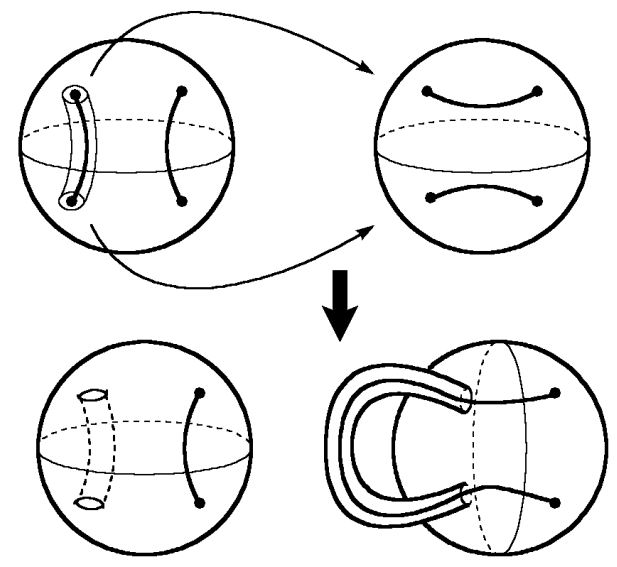

Figure 8.1.

Remark 8.1. In [17], Morimoto-Sakuma study the isotopy classes of the 1-bridge positions above. 
We say that these genus one 1-bridge positions are standard.

The purpose of this section is to prove:

Theorem 8.2. Every genus one 1-bridge positions of a non-trivial 2-bridge knot is standard.

First, we prepare some lemmas for the proof of Theorem 8.2, proofs of which are given in Appendix C. Let $\alpha$ be a trivial arc in a solid torus $T$. For $(T, \alpha)$, we have:

Lemma 8.3 (Appendix C-1). Let $D$ be an $\alpha$-compressing disk for $\partial T$. Then $D$ is either:

(1) a meridian disk of $T$ with $D \cap \alpha=\emptyset$. In this case, we obtain, by cutting $(T, \alpha)$ along $D$, a 1-string trivial tangle,

(2) a meridian disk of $T$ with $D \cap \alpha$ consists of one point, and we obtain, by cutting $(T, \alpha)$ along $D$, a 2-string trivial tangle, or

(3) $\partial$-parallel disk in $T$ with $D \cap \alpha=\emptyset$. In this case, $D$ cobounds a 1-string trivial tangle in $(T, \alpha)$.
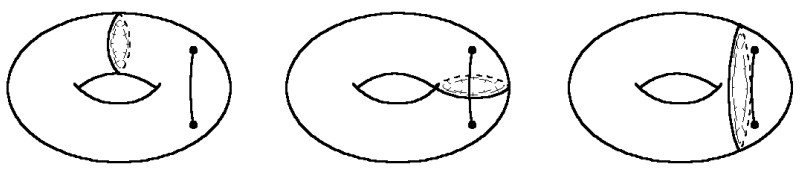

Figure 8.2.

Lemma 8.4 (Appendix C-2). Let $D$ be an $\alpha$-essential disk in $T$ such that $D \cap \alpha$ consists of two points. Then there exists an $\alpha$-compressing disk $D^{\prime}$ disjoint from $D$ such that $D^{\prime} \cap \alpha$ consists of one point. Moreover, by cutting $(T, \alpha)$ along $D^{\prime}$, we obtain 2 -string trivial tangle $(B, \beta)$ such that $D$ is a $\beta$-incompressible disk in $(B, \beta)$ (hence $D$ is $\beta$-boundary parallel).

We note that the disk $D$ in Lemma 8.4 is either separating or nonseparating in $T$.

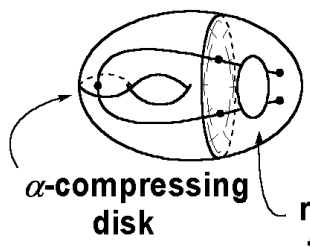

disk rational

tangle

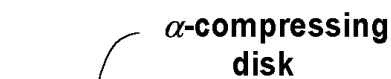

disk

rational

tangle

Figure 8.3.

Lemma 8.5 (Appendix C-3). Let $D_{1}, D_{2}$ be mutually disjoint non $\alpha$-parallel, $\alpha$-essential disks such that $D_{i} \cap \alpha(i=1,2)$ consists of two points. Then there exists an $\alpha$-compressing disk $D^{\prime}$ for $\partial T$ disjoint from $D_{1} \cup D_{2}$ such 
that $D^{\prime} \cap \alpha$ consists of one point. Moreover each $D_{i}$ is non-separating in $T$, and, by cutting $(T, \alpha)$ along $D^{\prime}$, we obtain 2 -string trivial tangle $(B, \beta)$, and $D_{1}, D_{2}$ are mutually non $\beta$-parallel, $\beta$-boundary parallel, $\beta$-incompressible disks in $(B, \beta)$.

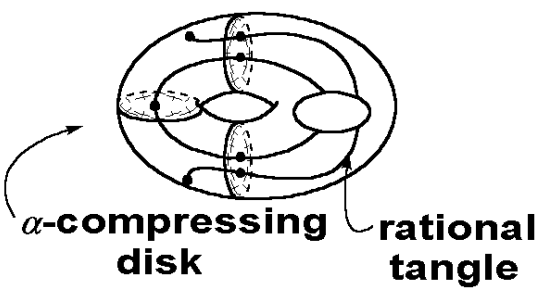

Figure 8.4.

Lemma 8.6 (Appendix C-4). Let $D$ be an $\alpha$-compressible disk such that $\partial D$ is $\alpha$-essential in $\partial T$, and $D \cap \alpha$ consists of two points. Then there is a disk $\Delta$ in $T$ such that $\Delta \cap D=\partial \Delta \cap D=\gamma$ an arc, and $\Delta \cap \alpha=c \ell(\partial \Delta-\gamma)$. Particularly, if $D$ is separating in $T$, then $D$ separates $(T, \alpha)$ into $\left(T^{\prime}, \alpha^{\prime}\right)$, and $\left(B^{\prime}, \alpha^{\prime \prime}\right)$ such that $\alpha^{\prime}$ is a trivial arc in a solid torus $T^{\prime}$. In this case, if $\left(B^{\prime}, \alpha^{\prime \prime}\right)$ happens to be a rational tangle, then $\left(B^{\prime}, \alpha^{\prime \prime}\right)$ is an $\alpha$-boundary parallelism.

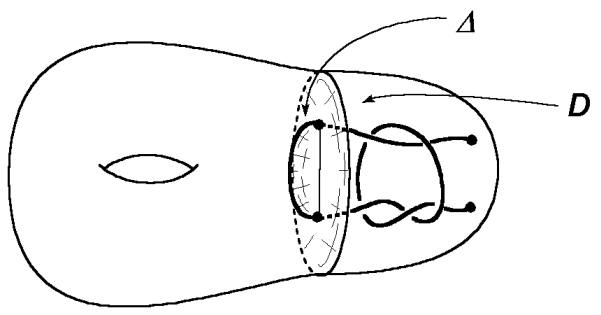

Figure 8.5.

In the rest of this section, we give a proof of Theorem 8.2. Let $K$ be a non-trivial 2-bridge knot. Let $A \cup_{P} B$ be a genus 0 Heegaard splitting of $S^{3}$ which gives a 2-bridge position of $K$, and $X \cup_{Q} Y$ a genus one Heegaard splitting which gives a genus one 1-bridge position of $K$. Note that $A \cup B$, $X \cup Y$ give orbifold Heegaard splittings of $\left(S^{3}, K\right)$ (see Example 6.11).

Proposition 8.7. Exactly one of the following (1) or (2) holds.

1. $X \cup_{Q} Y$ gives a standard genus one 1-bridge position.

2. $X \cup_{Q} Y$ is strongly $K$-irreducible.

Proof. Suppose that $X \cup_{Q} Y$ is weakly $K$-reducible. Let $D_{X}, D_{Y}$ be a pair of $K$-esssential disks in $X, Y$ respectively such that $\partial D_{X} \cap \partial D_{Y}=\emptyset$. Since $\mathrm{H}_{1}\left(S^{3}\right)=\{0\}$, we see that either $D_{X}$ is separating in $X$ or $D_{Y}$ is separating in $Y$. Without loss of generality, we may suppose that $D_{X}$ is separating in $X$. 
Claim 1. The disk $D_{Y}$ is non-separating in $Y$.

Proof. Assume that both $D_{X}$ and $D_{Y}$ are separating in $X$ and $Y$ respectively. By Lemma $8.3(3)$, we see that the closure of a component of $X-D_{X}$ $\left(Y-D_{Y}\right.$ resp. $)$, say $B_{X}^{3}\left(B_{Y}^{3}\right.$ resp. $)$, is a 3 -ball such that $K \cap B_{X}^{3}\left(K \cap B_{Y}^{3}\right.$ resp.) is a trivial arc. Since $\partial D_{X} \cap \partial D_{Y}=\emptyset$, we see that $\partial D_{X}$ and $\partial D_{Y}$ are $K$-parallel in $Q$. This implies that $B_{X}^{3} \cup B_{Y}^{3}$ is a 3 -ball, and $B_{X}^{3} \cap B_{Y}^{3}$ is a disk intersecting $K$ in two points. This shows that $K$ is a connected sum of trivial knots, and, hence, $K$ is a trivial knot, a contradiction.

Claim 2. The disk $D_{Y}$ intersects $K$ in one point.

Proof. Assume this does not hold, i.e., $D_{Y} \cap K=\emptyset$. Let $N\left(D_{Y}\right)$ be a regular neighborhood of $D_{Y}$ in $Y$. Let $X^{\prime}=X \cup N\left(D_{Y}\right)$, and $Y^{\prime}=c \ell\left(Y-N\left(D_{Y}\right)\right.$ ). Then, by Lemma $8.3(3)$, we see that $X^{\prime}$ is a 3 -ball such that $K \cap X^{\prime}$ is a trivial arc. Moreover, by Lemma 8.3 (1), we see that $Y^{\prime}$ is a 3 -ball such that $K \cap Y^{\prime}$ is a trivial arc. Hence we see that $K$ is a trivial knot, a contradiction.

Let $N^{\prime}\left(D_{Y}\right)$ be a regular neighborhood of $D_{Y}$ in $Y$. Let $X^{\prime \prime}=X \cup N^{\prime}\left(D_{Y}\right)$, and $Y^{\prime \prime}=c \ell\left(Y-N^{\prime}\left(D_{Y}\right)\right)$. Then, by Lemma $8.3(3)$, we see that $X^{\prime \prime}$ is a 3-ball such that $K \cap X^{\prime \prime}$ is a system of 2-string trivial arcs. Moreover, by Lemma 8.3 (2), we see that $Y^{\prime \prime}$ is a 3 -ball such that $K \cap Y^{\prime \prime}$ is a system of 2 -string trivial arcs. Hence $X^{\prime \prime} \cup Y^{\prime \prime}$ gives a 2-bridge position of $K$, and the genus one 1-bridge position $X \cup Y$ is obtained from $X^{\prime \prime} \cup Y^{\prime \prime}$ in a standard manner.

Conversely, suppose that $X \cup_{Q} Y$ gives a standard genus one 1-bridge position. Then it is clear there exist disks corresponding to $D_{X}, D_{Y}$ above in $X, Y$, and these disks give a weak $K$-irreducibility of $X \cup_{Q} Y$. This together with the above shows that $X \cup_{Q} Y$ gives a standard genus one 1-bridge position if and only if $X \cup_{Q} Y$ is weakly $K$-irreducible.

This completes the proof of Proposition 8.7.

Then we prove:

Proposition 8.8. Suppose that $P \cap Q$ consists of non-empty collection of simple closed curves which are $K$-essential in both $P$ and $Q$. Then the genus one 1-bridge position $X \cup_{Q} Y$ of $K$ is obtained from $A \cup_{P} B$ in a standard manner.

For the proof of Proposition 8.8, we prepare the following lemma, the proof of which is left to the reader.

Lemma 8.9. Let $T$ be a solid torus, and $A$ an annulus properly embedded in $T$ such that each component of $\partial A$ is a longitude of $T$. Then there is a homeomorphism $h:$ (annulus) $\times I \rightarrow T$ such that $h(($ annulus $) \times\{1 / 2\})=A$. 
Proof of Proposition 8.8. The proof is carried out by the induction of the number of the components $P \cap Q$. The following Claims 1 and 2 give the first step of the induction.

Claim 1. If $P \cap Q$ consists of one component, then the genus one 1-bridge position $X \cup_{Q} Y$ of $K$ is obtained from $A \cup_{P} B$ in a standard manner.

Proof. Let $D_{X}=P \cap X$, and $D_{Y}=P \cap Y$. Then $D_{X}, D_{Y}$ are disks properly embedded in $X, Y$ respectively, each of which intersect $K$ in two points such that $\partial D_{X}=\partial D_{Y}$. Since $P$ is separating in $S^{3}, D_{X}$ is separating in $X$, and this shows that $\partial D_{X}$ is contractible in $Q$. Let $E$ be the disk in $Q$ bounded by $\partial D_{X}\left(=\partial D_{Y}\right)$. Then $E$ intersects $K$ in two points. Without loss of generality, we may suppose that $E$ is contained in $A$. Since $E$ separates the boundary components of each component of $K \cap A$, we see that $E$ is $K$-incompressible in $A$. Then, by Lemma 7.3(2) we have either one of the following two cases.

Case 1. $E$ is not $K$-parallel to one of $D_{X}$ or $D_{Y}$ in $A$.

In this case, we may suppose without loss of generality that $E$ is not $K$ parallel to $D_{X}$. Then, by Lemma $7.3(2)$, we see that $E$ is $K$-parallel to $D_{Y}$ in $Y$, and, by Lemma 8.4, we see that there is a $K$-compressing disk $D$ for $\partial X$ in $X$ such that $D$ intersects $K$ in one point and $D \cap D_{X}=\emptyset$, and these imply that the genus one 1-bridge position $X \cup_{Q} Y$ of $K$ is $K$-isotopic to a genus one 1-bridge position which is obtained from $A \cup_{P} B$ in a standard manner by using the arc $K \cap(B \cap X)$.

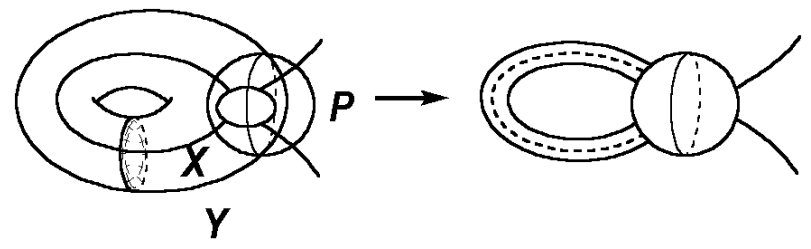

Figure 8.6.

Case 2. $E$ is $K$-parallel to $D_{X}$ and $D_{Y}$ in $A$.

In this case, we consider the genus one surface $F=Q \cap B$. Note that $\partial F$ is a $K$-essential simple closed curve in $P$. Then, by Lemma 7.3, we see that there is a $K$-compressing disk $D$ for $F$ in $B$. Without loss of generality, we may suppose that $D$ is contained in $X$. Then we have the following two cases.

Case 2.1. $D \cap K=\emptyset$.

In this case, we obtain a $K$-compressing disk $D^{\prime}$ for $\partial B$ in $B$ by compressing $F$ along $D$. By applying a slight isotopy, we may regard $D^{\prime}$ as a 
$K$-compressing disk for $\partial X$ in $X$ such that $\partial D^{\prime}=\partial E$. Then, by Lemma 8.6, we see that the arc $K \cap X$ is pushed into by an isotopy rel $\partial$ in $X$ to an arc contained in $E$. Then we further push the arc along the parallelism between $E$ and $D_{Y}$ to an arc contained in the disk $D_{Y}$. We denote by $K^{\prime}$ the image of $K$ under this isotopy. Then $K^{\prime}$ is contained in $B$ and $K^{\prime} \cap \partial B$ is an arc. Since this isotopy does not move the component of $K \cap B$ contained in $Y$ $\left(=c \ell\left(K^{\prime} \cap \operatorname{Int} B\right)\right), c \ell\left(K^{\prime} \cap \operatorname{Int} B\right)$ is a trivial arc in $B$. This implies that $K$ is a trivial knot, a contradiction.

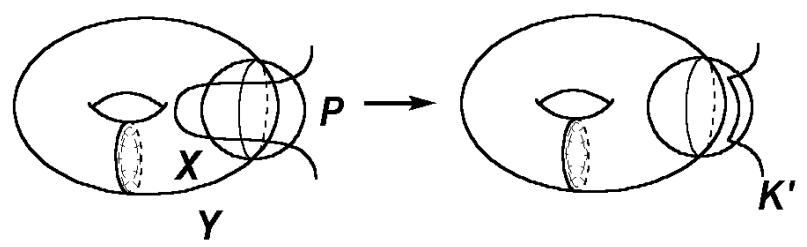

Figure 8.7.

Case 2.2. $D \cap K$ consists of one point.

In this case, we obtain, by applying $K$-compression on $F$ along $D$ and slight isotopy, a disk $D^{\prime \prime}$ in $B$ with $\partial D^{\prime \prime}=\partial E$, and $D^{\prime \prime}$ intersects $K$ in two points.

Subclaim. $D^{\prime \prime} \cup E$ bounds a $K$-parallelism between $D^{\prime \prime}$ and $E$ in $X$.

Proof. Assume not. Suppose that $D^{\prime \prime}$ is $K$-compressible in $X$. Then, by applying $K$-compression and slight isotopy, we obtain a $K$-compressing disk $E^{\prime}$ in $B$ such that $\partial E^{\prime}=\partial E$. Then, by the argument of Case 2.1 , we see that $K$ is a trivial knot, a contradiction. Suppose that $D^{\prime \prime}$ is $K$-incompressible in $X$. By the assumption, we see that $E^{\prime} \cup D_{X}$ bounds a rational tangle in $X$ which is not a $K$-parallelism between $E^{\prime}$ and $D_{X}$. Then, by Lemma 7.3 (2), we see that $E^{\prime}$ and $D_{Y}$ must bound a $K$-parallelism in $B$. But this is impossible, since $D_{Y}$ contains the boundary components of a component of $K \cap B$.

By the subclaim together with the arguments in Case 1, we see that the genus one 1-bridge position $X \cup_{Q} Y$ of $K$ is $K$-isotopic to a genus one 1bridge position which is obtained from $A \cup_{P} B$ in a standard manner by using the arc $K \cap(B \cap X)$. 


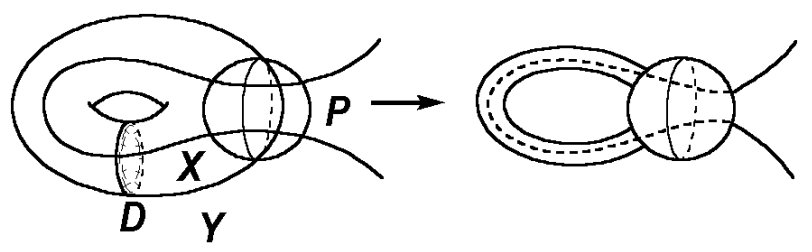

Figure 8.8.

This completes the proof of Claim 1.

Claim 2. If $P \cap Q$ consists of two components, then the genus one 1-bridge position $X \cup_{Q} Y$ of $K$ is obtained from $A \cup_{P} B$ in a standard manner.

Proof. Let $D_{1}, A_{0}, D_{2}$ be the closures of the components of $P-(P \cap Q)$ such that $D_{1}$ and $D_{2}$ are disks, and $A_{0}$ is an annulus. Without loss of generality, we may suppose that $D_{1} \cup D_{2}$ is contained in $X$, and $A_{0}$ is contained in $Y$. Since $P$ is separating in $S^{3}$, we see that both $D_{1}$ and $D_{2}$ are either separating or non-separating in $X$.

Case 1. Both $D_{1}$ and $D_{2}$ are separating in $X$.

Let $E$ be the disk in $Q$ bounded by $\partial D_{1}$. By changing subscript, if necessary, we may suppose that $\partial D_{2} \cap E=\emptyset$, i.e., $D_{1}$ is "outer" than $D_{2}$. Without loss of generality, we may suppose that $E$ is contained in $A$. Then we have the following cases.

Case 1.1. $D_{1}$ and $E$ are $K$-parallel in $X$.

In this case, we can push $D_{1}$ along the parallelism out of $X$ to make $P \cap X=D_{2}$. Hence, we have the conclusion by Claim 1 .

Case 1.2. $D_{1}$ and $E$ are not $K$-parallel in $X$.

In this case, we first claim that $D_{1}$ is $K$-incompressible in $X$. Assume that $D_{1}$ is $K$-compressible in $X$. Then, by Lemma 8.6, the component of $K-D_{1}$ contained in $X$ is isotopic rel $\partial$ to an arc in $D_{1}$ joining $D_{1} \cap K$. We denote by $K^{\prime}$ the image of $K$ under this isotopy. Then $K^{\prime}$ is contained in $A$ and $K^{\prime} \cap \partial A\left(=K^{\prime} \cap D_{1}\right)$ is an arc, and $c \ell\left(K^{\prime} \cap \operatorname{Int} A\right)$ is a trivial arc in $A$ (see Claim 1, Case 2.1). This implies that $K$ is a trivial knot, a contradiction. Hence $D_{1}$ is $K$-incompressible in $X$.

Let $B^{3}$ be the 3 -ball in $X$ bounded by $D_{1} \cup E$. By Lemma 8.4, we see that $\left(B^{3}, K \cap B^{3}\right)$ is a rational tangle. Assume that $E$ is $K$-compressible in $A$. Then by applying the last half of Lemma 7.4 to $E$ in $A$, we see that $\left(B^{3}, K \cap B^{3}\right)$ is a $K$-parallelism, contradicting the fact that $D_{1}$ and $E$ are not $K$-parallel in $X$. Hence $E$ is $K$-incompressible in $A$. Then, by Lemma 7.3 (2), we see that $E$ and $c \ell\left(P-D_{1}\right)$ bounds a $K$-parallelism in $A$. However 
this is impossible, since the boundary components of a component of $K \cap A$ is contained in $c \ell\left(P-D_{1}\right)$. This shows that Case 1.2 does not occur.

Case 2. Both $D_{1}$ and $D_{2}$ are non-separating in $X$.

In this case, we first note that, since $D_{i} \cap(X \cap K)(i=1,2)$ consists of two points, the two points $K \cap Q$ are contained in a component of $Q-\left(\partial D_{1} \cup \partial D_{2}\right)$. Then, let $R$ be the closure of the component of $X-\left(D_{1} \cup D_{2}\right)$ which does not contain $K \cap Q$. Without loss of generality, we may suppose that $R$ is contained in $A$. We have the following cases.

Case 2.1. Both $D_{1}$ and $D_{2}$ are $K$-incompressible in $X$.

In this case, we have the following subcases.

Case 2.1.1. $D_{1}$ and $D_{2}$ are not $K$-parallel in $X$.

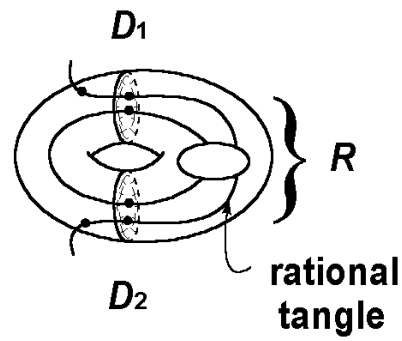

Figure 8.9.

By Lemma 8.5, we see that $(R, K \cap R)$ is a rational tangle. By Lemma 8.9, we see that $A_{0}$ is parallel to the annulus $R \cap Q$ in $Y$, and, hence, $P$ is $K$ isotopic to $\partial R$. Now let $a$ be the component of $K \cap B$ that is contained in $X$. Then, by Lemma 8.5 again, we see that the torus obtained from $\partial R$ by adding a tube along $a$ is $K$-isotopic to $Q$. Hence we have seen that the genus one 1-bridge position $X \cup_{Q} Y$ of $K$ is obtained from $A \cup_{P} B$ in a standard manner by using the arc $a$.

Case 2.1.2. $D_{1}$ and $D_{2}$ are $K$-parallel in $X$, and there exists a $K$-incompressible disk $D^{\prime}$ in $X$ such that $D^{\prime}$ intersects $K$ in two points, $D^{\prime}$ is nonseparating in $X, D^{\prime} \cap\left(D_{1} \cup D_{2}\right)=\emptyset$, and $D^{\prime}$ is not $K$-parallel to $D_{1}$ (or $\left.D_{2}\right)$.

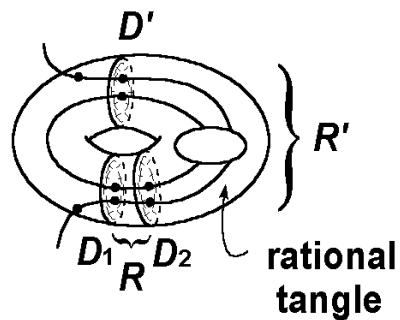

Figure 8.10. 
Let $R^{\prime}$ be the closure of the component of $X-\left(R \cup D^{\prime}\right)$ which does not contain $K \cap Q$. By exchanging subscript, if necessary, we may suppose that $R^{\prime} \cap R=D_{2}$. Let $A^{\prime}=R^{\prime} \cap Q$, and $D^{\prime \prime}=A^{\prime} \cup D^{\prime}$. We note that $D^{\prime \prime}$ is a disk properly embedded in $B$, which intersects $K$ in two points. Since $D^{\prime \prime}$ separates the boundary components of each component of $K \cap B$ in $P$, we see that $D^{\prime \prime}$ is $K$-incompressible. Moreover $D^{\prime \prime}$ and $D_{2}$ are not $K$-parallel in $X$, hence in $B$. Hence, by Lemma $7.3(2)$, we see that $D^{\prime \prime}$ and $\mathrm{c} \ell\left(P-D_{2}\right)$ $\left(=D_{1} \cup A_{0}\right)$ are $K$-parallel in $B$. This shows that $P$ is $K$-isotopic to $\partial R^{\prime}$. Then, by the argument of Case 2.1.1, with regarding $R^{\prime}$ as $R$ we see that the genus one 1-bridge position $X \cup_{Q} Y$ of $K$ is obtained from $A \cup_{P} B$ in a standard manner.

Case 2.1.3. $D_{1}$ and $D_{2}$ are $K$-parallel in $X$, and there does not exist a $K$-incompressible disk $D^{\prime}$ as in Case 2.1.2.

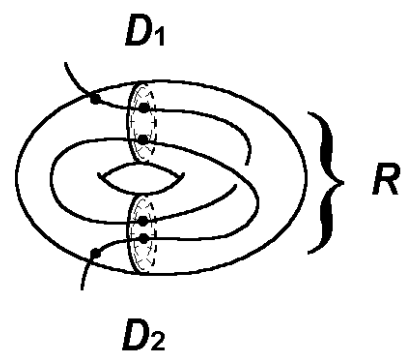

Figure 8.11.

In this case, it is easy to see that the argument of Case 2.1.1 works to show that the genus one 1-bridge position $X \cup_{Q} Y$ of $K$ is obtained from $A \cup_{P} B$ in a standard manner.

Case 2.2. Either $D_{1}$ or $D_{2}$ is $K$-compressible in $X$.

Without loss of generality, we may suppose that there is a $K$-compressing disk $\mathcal{D}$ for $D_{1} \cup D_{2}$ such that $\partial \mathcal{D} \subset D_{2}$.

By applying $K$-compression on $D_{2}$, we obtain a compressing disk $D$ for $\partial X$ in $X$ such that $D \cap K=\emptyset$. By applying a slight isotopy, we may suppose that $D_{2} \cap D=\emptyset$. By Lemma 8.9, we see that $A_{0}$ and $R \cap Q$ are parallel in $Y$. Hence, by $K$-isotopy, we may suppose that $P=\partial R$, (and $A=R$ ).

Then we have the following subcases.

Case 2.2.1. $D$ is not contained in $R$. 


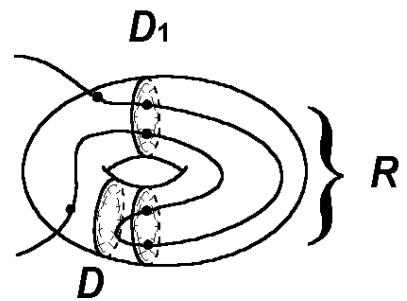

$D_{2}$

Figure 8.12.

Since $\partial D_{1}$ and $\partial D_{2}$ are $K$-parallel, we see that $D_{1}$ is also $K$-compressible in $X$. Then, by Lemma 8.6, we see that there is a disk $\Delta$ in $X$ such that $\Delta \cap D_{1}=\partial \Delta \cap D_{1}=\gamma$ an arc, and $\Delta \cap(K \cap X)=c \ell(\partial \Delta-\gamma)$. Hence, we can move $K$ by an isotopy along $\Delta$ such that $\Delta \cap(K \cap X)$ is moved to $\gamma$ (hence, the component of $K \cap B$ which intersects $Y$ is not changed by this isotopy). Since each component of $K \cap B$ is a trivial arc, this shows that $K$ is a trivial knot, a contradiction.

Case 2.2.2. $D$ is contained in $R$.

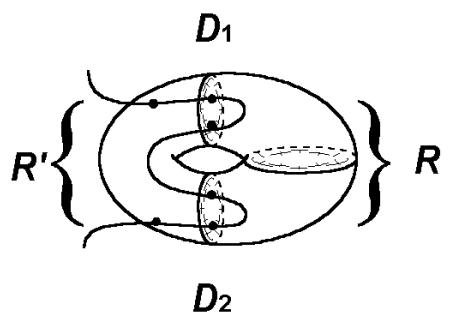

Figure 8.13.

Let $R^{\prime}=\mathrm{c} \ell(X-R)$, and $A^{*}=R^{\prime} \cap \partial X$. Then $A^{*}$ is an annulus properly embedded in $B$ such that each component of $\partial A^{*}$ is $K$-essential in $\partial B$, and $A^{*}$ intersects $K$ in two points. By Lemma 7.3, we see that there is a $K$-compressing disk $D^{\prime}$ for $A^{*}$ in $B$.

Then we claim that $D^{\prime}$ is contained in $X$. In fact, assume that $D^{\prime}$ is contained in $Y$. Since $H_{1}\left(S^{3}\right)=\{0\}$, we see that $\partial D^{\prime}$ bounds a disk $E$ in $A^{*}$ such that $E \cap K$ consists of two points, and $D^{\prime} \cap K=\emptyset$. Let $B^{3 \prime}$ be the 3-ball in $Y$ bounded by $D^{\prime} \cup E$, and $B^{3}=\mathrm{c} \ell(X-N(D))$. By Lemma 8.3 (1), we see that $K \cap B^{3}(=K \cap X)$ is a trivial arc in $B^{3}$. By Lemma 8.3 (3), we see that $K \cap B^{3 \prime}$ is a trivial arc in $B^{3 \prime}$. Note that $B^{3} \cup B^{3 \prime}$ is a 3-ball, and $B^{3} \cap B^{3 \prime}=E$. This shows that $K$ is a trivial knot, a contradiction.

Hence $D^{\prime}$ is contained in $X$. Since each component of $\partial A^{*}$ separates the boundary points of each component of $K \cap B$ in $P$, we see that $D^{\prime} \cap K \neq \emptyset$, 
and, hence, $D^{\prime} \cap K$ consists of a point. By applying $K$-compression on $A^{*}$ along $D^{\prime}$, we obtain two disks $D_{1}^{\prime}, D_{2}^{\prime}$ in $B$ such that $\partial D_{1}^{\prime}=\partial D_{1}$, and $\partial D_{2}^{\prime}=\partial D_{2}$. Since $D_{i}^{\prime}(i=1,2)$ separates the boundary components of a component of $K \cap B, D_{i}^{\prime}$ is $K$-incompressible in $B$. Then, by Lemma 7.3 (2), $D_{i} \cup D_{i}^{\prime}(i=1,2)$ bounds a rational tangle in $B$. Let $B^{3 \prime \prime}$ be the 3 -ball obtained from $X$ by cutting along $D^{\prime}$. We regard $D_{1}^{\prime}, D_{2}^{\prime}$ as contained in $\partial B^{3 \prime \prime}$, and $D_{1}, D_{2}$ are properly embedded in $B^{3 \prime \prime}$. By Lemma $8.3(2)$, we see that $\left(B^{3 \prime \prime}, K \cap B^{3 \prime \prime}\right)$ is a 2-string trivial tangle. Then we see that $D_{1}, D_{2}$ are $K$-compressible, by the condition of Case 2.2. Hence, by the last half of Lemma 7.4, we see that $D_{1}$ and $D_{1}^{\prime}\left(D_{2}\right.$ and $D_{2}^{\prime}$ resp. $)$ are $K$-parallel. Let $a$ be the component of $K \cap B$ that is contained in $X$. Then, by the above observations, we see that the genus one 1-bridge position $X \cup_{Q} Y$ of $K$ is obtained from $A \cup_{P} B$ in a standard manner by using $a$.

This completes the proof of Claim 2.

Completion of the proof of Proposition 8.8. Suppose that $\sharp(P \cap Q) \geq 3$. Note that the components of $P \cap Q$ are mutually $K$-parallel in $P$. Let $D_{1}$, $A_{1}, \ldots, A_{m}, D_{2}$ be the closures of the components of $P-(P \cap Q)$ such that $D_{1}, D_{2}$ are disks and $A_{1}, \ldots, A_{m}$ are annuli that are located on $P$ in this order. Then we have the following cases.

Case 1. Either $D_{1}$ or $D_{2}$ is non-separating in the solid torus.

Without loss of generality, we may suppose that $D_{1}$ is contained in $X$, and is non-separating in $X$. Then $A_{1}$ is contained in $Y$, and, by Lemma 8.9, there is a homeomorphism from $A_{1} \times I$ to $Y$ such that $A_{1}$ corresponds to $A_{1} \times\{1 / 2\}$. Let $U$ be the closure of the component of $Y-A_{1}$ which does not contain $K \cap Q$.

Suppose that $(\operatorname{Int} U) \cap P \neq \emptyset$. Then we can push the component of (Int $U) \cap P$ along the parallelism $U$ to $X$ to reduce $\sharp(P \cap Q)$, yet still have at least two components $\partial A_{1}$.

Suppose that $(\operatorname{Int} U) \cap P=\emptyset$. Then we can push $A_{1}$ along the parallelism $U$ to $X$ to reduce $\sharp(P \cap Q)$ by two.

In either case we have the conclusion by the assumption of the induction. Case 2. Both $D_{1}$ and $D_{2}$ are separating in the solid torus.

If $D_{1}$ or $D_{2}$ is $K$-boundary parallel, then we can apply the assumption of the induction by the argument as in Case 1 . Hence we suppose that $D_{1}$ and $D_{2}$ are not $K$-boundary parallel. Let $E_{i}$ be the disk in $Q$ bounded by $\partial D_{i}$. Without loss of generality, we may suppose that $E_{1} \subset E_{2}$, and $D_{1}$ is contained in $X$. Then we have the following subcases.

Case 2.1. $D_{1}$ is $K$-incompressible in $X$.

Let $B^{3}$ be the closure of the component of $X-D_{1}$ such that $\partial B^{3}=$ $D_{1} \cup E_{1}$. Then, by Lemma $8.4,\left(B^{3}, K \cap B^{3}\right)$ is a rational tangle. Suppose 
$\left(\operatorname{Int} B^{3}\right) \cap P \neq \emptyset$. We note that each component of $c \ell\left(\left(\operatorname{Int} B^{3}\right) \cap P\right)$ is an annulus whose boundary components are parallel to $\partial D_{1}$ in $Q-K$. This shows that each annulus is $K$-incompressible in $B^{3}$. Hence, by Lemma 7.3 (3), we see that the closure of each component of $\left(\operatorname{Int} B^{3}\right) \cap P$ is boundary parallel annulus. Hence, we can push them to $Y$ to reduce $\sharp(P \cap Q)$, and we have the conclusion by the assumption of the induction. If $\left(\operatorname{Int} B^{3}\right) \cap P=\emptyset$, then by Lemma $7.3(2)$, the last half of Lemma 7.4, and the assumption that $D_{1}$ is not $K$-boundary parallel in $X$, we see that $\mathrm{c} \ell\left(P-D_{1}\right)$ is $K$-isotopic to $E_{1}$ rel $D_{1}$. Hence, by the argument in Claim 1 , we see that the genus one 1-bridge position $X \cup_{Q} Y$ of $K$ is obtained from $A \cup_{P} B$ in a standard manner.

Case 2.2. $D_{1}$ is $K$-compressible in $X$.

We moreover have the following subcases.

Case 2.2.1. $D_{2} \subset Y$, and $D_{2}$ is $K$-incompressible in $Y$.

Let $B^{3}$ be the closure of the component of $Y-D_{2}$ such that $\partial B^{3}=D_{2} \cup E_{2}$. In this case, since $\partial D_{1} \subset \operatorname{Int} E_{2}$, we see that $\left(\operatorname{Int} B^{3}\right) \cap P \neq \emptyset$. Hence, by the argument of Case 2.1 (for the case $\left(\operatorname{Int} B^{3}\right) \cap P \neq \emptyset$ ), we can show that the genus one 1-bridge position $X \cup_{Q} Y$ of $K$ is obtained from $A \cup_{P} B$ in a standard manner.

Case 2.2.2. $D_{2} \subset Y$, and $D_{2}$ is $K$-compressible in $Y$.

In this case, by $K$-compressing $D_{2}$, we obtain a $K$-compressing disk $D_{2}^{\prime}$ for $\partial Y$ in $Y$ such that $\partial D_{2}^{\prime}=\partial D_{2}$. Then, by Lemma $8.3(3)$, we see that $K \cap Y$ is rel $\partial$ isotopic to an $\operatorname{arc} \alpha_{Y}$ in $E_{1}$. And we also see that $K \cap X$ is rel $\partial$ isotopic to an arc $\alpha_{X}$ in $E_{1}$ such that $\alpha_{X} \cap \alpha_{Y}=\partial \alpha_{X}=\partial \alpha_{Y}$. Hence $K$ is a trivial knot, a contradiction.

Case 2.2.3. $D_{2} \subset X$.

Let $T^{\prime}$ and $B^{3}$ be the closures of the component of $T-D_{1}$ such that $T^{\prime}$ is a solid torus and $B^{3}$ is a 3 -ball. Without loss of generality, we may suppose that $B^{3}$-side of $D_{1}$ is contained in $B$. Since $D_{1}$ is $K$-compressible, we see that $K \cap T^{\prime}$ is rel $\partial$ isotopic in $T^{\prime}$ to an arc $\alpha$ in $D_{1}$, by an isotopy that does not move $K-T^{\prime}$. Note that since $D_{2} \subset X, \mathrm{c} \ell\left(K-T^{\prime}\right)$ is a component of $K \cap B$, hence, a trivial arc in $B$. This shows that $K$ is a trivial knot, a contradiction.

This completes the proof of Proposition 8.8.

Proof of Theorem 8.2. Let $A \cup_{P} B$ be a Heegaard splitting which gives a 2bridge position of $K$, and $X \cup_{Q} Y$ a Heegaard splitting which gives the given genus one 1-bridge position of $K$. By Proposition 8.7, it is enough to assume that $X \cup_{Q} Y$ is strongly $K$-irreducible for the proof of Theorem 8.2. Then, by Proposition 7.5, and Corollary 6.22, we may suppose that $P$ and $Q$ intersect 
in non-empty collection of simple closed curves which are $K$-essential in both $P$ and $Q$. Then we have the conclusion by Proposition 8.8.

\section{Appendix A.}

Let $\gamma$ be a system of trivial arcs in a handlebody $H$, and $p: \tilde{H} \rightarrow H$ the two fold branched cover of $H$ along $\gamma$.

Let $F$ be a surface properly embedded in $H$, which is in general position with respect to $\gamma$. Then, by using $\mathbb{Z}_{2}$-equivariant loop theorem [12], we see that:

Lemma A.1. $F$ is $\gamma$-incompressible if and only if $\tilde{F}\left(=p^{-1}(F)\right)$ is incompressible.

Moreover, by using $\mathbb{Z}_{2}$-equivariant cut and paste argument as in [9, Proof of 10.3], we see that:

Lemma A.2. A $\gamma$-incompressible surface $F$ is $\gamma$-boundary compressible if and only if $\tilde{F}$ is boundary compressible.

By using $\mathbb{Z}_{2}$-Smith conjecture ([21], [16]) together with the $\mathbb{Z}_{2}$-equivariant cut and paste argument and the irreducibility of $H$, we have:

Lemma A.3. A $\gamma$-incompressible surface $F$ is $\gamma$-boundary parallel if and only if $\tilde{F}$ is boundary parallel. In particular, if $F$ is a disk intersecting $\gamma$ in one point, and $\partial F$ bounds a disk $D$ in $\partial H$ such that $D$ intersects $\gamma$ in one point, then $F$ is $\gamma$-boundary parallel (in fact, $F$ and $D$ are $\gamma$-parallel).

\section{Appendix B.}

Let $(B, \beta)$ be a 2 -string trivial tangle, and $(\tilde{B}, \tilde{\beta})$ the 2 -fold branched covering space of $B$ along $\beta$. Then $\tilde{B}$ is a solid torus, $\tilde{\beta}$ a system of two trivial $\operatorname{arcs}$ in $\tilde{B}$, and the covering translation $\tau$ is a $\pi$-rotation along $\tilde{\beta}$ (for details, see [3, Chapter 12]).

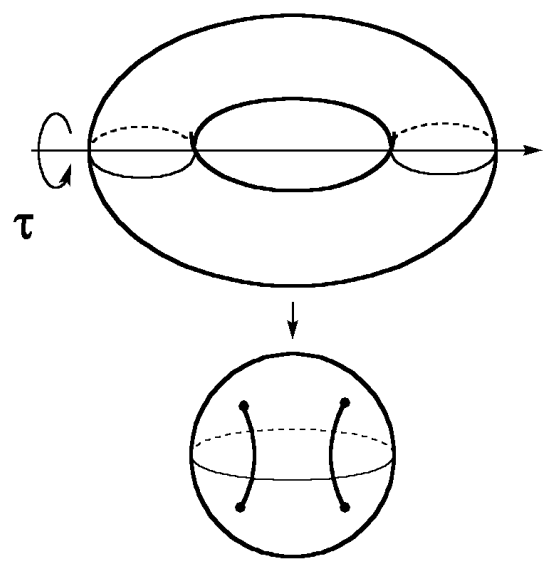

Figure B-1. 
We leave the proof of the next lemma to the reader.

Lemma B.0. Let $F$ be an orientable incompressible surface properly embedded in a solid torus. Then either:

1. $F$ is a meridian disk,

2. $F$ is a boundary parallel disk, or

3. $F$ is a boundary parallel annulus.

Then, we show:

Lemma B.1. Let $D$ be a $\beta$-essential surface in $B$. Then $D$ is a disk disjoint from $\beta$, and $D$ separates the components of $\beta$.

Proof. Let $\tilde{D}$ be the lift of $D$ in $\tilde{B}$. By Lemmas A-1, A-3, we see that $\tilde{B}$ is an essential surface in the solid torus $\tilde{B}$. By Lemma B-0, we see that $\tilde{D}$ is a meridian disk. Suppose that $\tilde{D} \cap \tilde{\beta} \neq \emptyset$. Then we see that $\tilde{D} \cap \beta$ consists of a point, and, hence, $D \cap \beta$ consists of a point. However this implies that $\partial D$ is $\beta$-inessential in $\partial B$. Hence, by Lemmas $\mathrm{A}-3$, and $\mathrm{B}-0$, we see that $D$ is $\beta$-boundary parallel, a contradiction. Since $\partial D$ is $\beta$-essential in $\partial B$, we see that $\partial D$ separates the points $\partial \beta$ in $\partial B$. This shows that $D$ separates the components of $\beta$.

Lemma B.2. Let $F$ be a $\beta$-incompressible surface in $B$.

Then either (0) $F$ is $\beta$-essential, (1) $F$ is a $\beta$-boundary parallel disk intersecting $\beta$ in at most one point, (2) $F$ is a $\beta$-boundary parallel disk intersecting $\beta$ in two points and $F$ separates $(B, \beta)$ into the parallelism and a rational tangle, or (3) $F$ is a $\beta$-boundary parallel annulus such that $F \cap \beta=\emptyset$.

Proof. Let $\tilde{F}$ be the lift of $F$ in $\tilde{B}$. By Lemma A-1, we see that $\tilde{F}$ is one of (1), (2), or (3) of Lemma B-0. It is easy to see that (1) ((2) resp.) of Lemma B-0 corresponds to the conclusion (0) ((1) resp.). Suppose that $\tilde{F}$ is an incompressible annulus ((3) of Lemma B-0). Then it is easy to see that we have conclusion (2) if $\tilde{F} \cap \tilde{\beta} \neq \emptyset$, and we have conclusion (3) if $\tilde{F} \cap \tilde{\beta}=\emptyset$.

Lemma B.3. Let $D$ be a $\beta$-compressible disk in $B$ such that $\partial D$ is $\beta$ essential in $\partial B$, and $D \cap \beta$ consists of two points.

Then $D$ separates $(B, \beta)$ into two tangles $\left(B_{1}, \beta_{1}\right)$, and $\left(B_{2}, \beta_{2}\right)$, where $\left(B_{1}, \beta_{1}\right)$ is a rational tangle such that there is a $\beta$-essential disk $D^{\prime}$ in $\left(B_{1}, \beta_{1}\right)$ with $D \cap D^{\prime}=\emptyset$. Moreover if $\left(B_{2}, \beta_{2}\right)$ happens to be a rational tangle, then $\left(B_{2}, \beta_{2}\right)$ is a $\beta$-boundary parallelism for $D$.

Proof. Let $\tilde{D}$ be the lift of $D$ in $\tilde{B}$. By Lemma A-1, we see that $\tilde{D}$ is a compressible annulus in $\tilde{B}$. Since $\partial D$ is $\beta$-essential, we see that, by compressing $\tilde{D}$, we obtain two meridian disks, say $D_{1}$ and $D_{2}$. Let $B_{1}^{3}, B_{2}^{3}$ be the closures of the components of $\tilde{B}-\left(D_{1} \cup D_{2}\right)$. Then $B_{1}^{3}, B_{2}^{3}$ are 3 -balls, 
and the closure of a component of $\tilde{B}-\tilde{D}$, say $\tilde{T}$, is obtained from one of $B_{1}^{3}$ or $B_{2}^{3}$, say $B_{1}^{3}$, by adding a 1 -handle, and hence $\tilde{T}$ is a solid torus, and there is an equivariant compressing disk $\tilde{D}^{\prime}$ for $\tilde{D}$ in $\tilde{T}$ such that $\tau\left(\tilde{D}^{\prime}\right) \cap \tilde{D}^{\prime}=\emptyset$. Hence $\tilde{T} / \tau$ gives a rational tangle, and $p\left(\tilde{D}^{\prime}\right)$ gives $D^{\prime}$. Then the closure of the other component of $\tilde{B}-\tilde{D}$, say $E$, is obtained from $B_{2}^{3}$ by removing a regular neighborhood of an arc properly embedded in $B_{2}^{3}$, and taking the closure. This shows that $E$ is homeomorphic to the exterior of a knot in $S^{3}$ by a homeomorphism such that $E \cap \partial T$ is a regular neighborhood of a meridian loop of the knot in $\partial E$. Suppose that $E$ is a solid torus, i.e., $(E, K \cap E)$ is a rational tangle. Then the knot is a trivial knot. Since $E \cap \partial T$ is a regular neighborhood of a meridian loop of the knot, we see that $E$ is a $\partial$-parallelism for $\tilde{D}$, and by Lemma A-3, we see that $\left(B_{2}, \beta_{2}\right)$ is a $\beta$-boundary parallelism for $D$.

\section{Appendix C.}

Let $\alpha$ be a trivial arc in a solid torus $T$, and $(\tilde{T}, \tilde{\alpha})$ the 2 -fold branched covering space of $T$ along $\alpha$. Then $\tilde{T}$ is a genus two handlebody, $\tilde{\alpha}$ a $1-$ string trivial arc in $\tilde{T}$, and the covering translation is a $\pi$-rotation along $\tilde{\alpha}$.

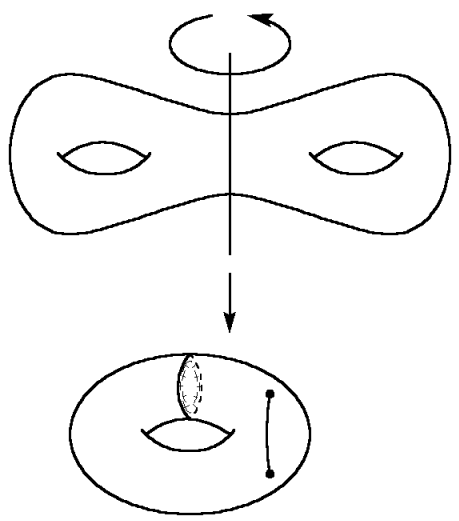

Figure C-1.

Lemma C.1. Let $D$ be an $\alpha$-compressing disk for $\partial T$. Then $D$ is either:

(1) a meridian disk of $T$ with $D \cap \alpha=\emptyset$. In this case, we obtain, by cutting $(T, \alpha)$ along $D$, a 1-string trivial tangle,

(2) a meridian disk of $T$ with $D \cap \alpha$ consists of one point, and we obtain by cutting $(T, \alpha)$ along $D$, a 2-string trivial tangle, or

(3) $\partial$-parallel disk in $T$ with $D \cap \alpha=\emptyset$. In this case, $D$ cobounds a 1-string trivial tangle in $(T, \alpha)$. 
Proof. Let $\tilde{D}$ be the lift of $D$ in $\tilde{T}$. Then we have either $\tilde{D}$ is a union of two disks if $D \cap \alpha=\emptyset$ (Case 1), or $\tilde{D}$ is a disk if $D \cap \alpha$ consists of one point (Case 2).

In Case 1 , we have either each component of $\tilde{D}$ is non-separating, or separating, which correspond to the conclusions (1), (3) respectively. In Case 2 , it is easy, by a homological argument, to see that $\tilde{D}$ is non-separating, and this gives conclusion (2)

Lemma C.2. Let $D$ be an $\alpha$-essential disk in $T$ such that $D \cap \alpha$ consists of two points. Then there exists an $\alpha$-compressing disk $D^{\prime}$ for $\partial T$ such that $D^{\prime} \cap D=\emptyset$, and $D^{\prime} \cap \alpha$ consists of one point. Moreover, by cutting $(T, \alpha)$ along $D^{\prime}$, we obtain a 2-string trivial tangle $(B, \beta)$ such that $D$ is a $\beta$-incompressible disk in $(B, \beta)$ (hence $D$ is $\beta$-boundary parallel).

Proof. Let $\tilde{D}$ be the lift of $D$ in $\tilde{T}$. By Lemma A-1, we see that $\tilde{D}$ is an essential annulus in $\tilde{T}$. Then it is easy to see that:

The annulus $\tilde{D}$ is obtained from a meridian disk $\Delta$ by attaching a band. (For a proof of this, see, for example, [13, Lemma 3.2].)

Let $\tilde{F}=c \ell(\partial \tilde{T}-N(\partial \tilde{D})$, where $N(\partial \tilde{D})$ is a regular neighborhood of $\partial \tilde{D}$ in $\partial \tilde{T}$. Note that $\tilde{F}$ is compressible in $\tilde{T}$ (in fact, slightly push off of $\Delta$ gives a compressing disk of $\tilde{F}$ ). Hence, by $\mathbb{Z}^{2}$-equivariant loop theorem [12], we have an equivariant compressing $\operatorname{disk}(\mathrm{s}) G$ for $\tilde{F}$.

Claim 1. $G$ consists of one disk, and, hence, $G \cap \alpha$ consists of one point.

Proof. Assume that $G$ consists of two disks $D_{1}, D_{2}$. Then we have the following three cases.

Case 1. Each $D_{i}$ is separating in $\tilde{T}$.

In this case $D_{1}$ and $D_{2}$ are parallel and the closures of $\tilde{T}-G$ are two solid tori $T_{1}, T_{2}$, and a 3 -ball $B$, which is a parallelism between $D_{1}$, and $D_{2}$. Note that $\tilde{\alpha}$ is contained in $B$, and this shows that $\tilde{D}$ is contained in $B$, contradicting the incompressibility of $\tilde{D}$.

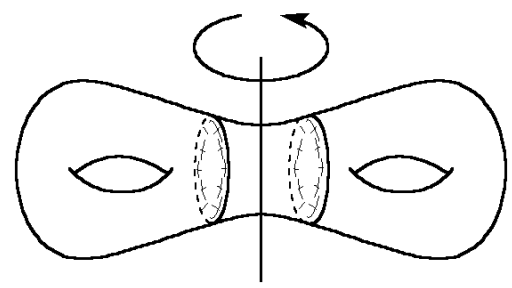


Case 2. Each $D_{i}$ is non-separating, and $G=D_{1} \cup D_{2}$ is non-separating in $\tilde{T}$.

Since $G \cap \tilde{D}=\emptyset$, this contradicts the incompressibility of $\tilde{D}$.

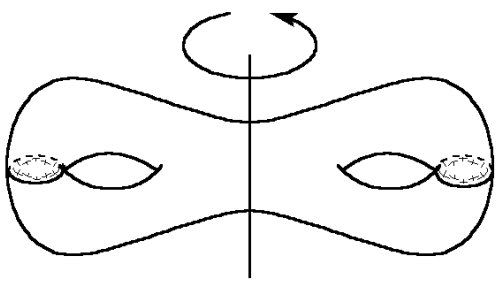

Figure C-3.

Case 3. Each $D_{i}$ is non-separating, and $G=D_{1} \cup D_{2}$ is separating in $\tilde{T}$.

In this case, we see that $D_{i}(i=1,2)$ intersects $\tilde{\beta}$, a contradiction.

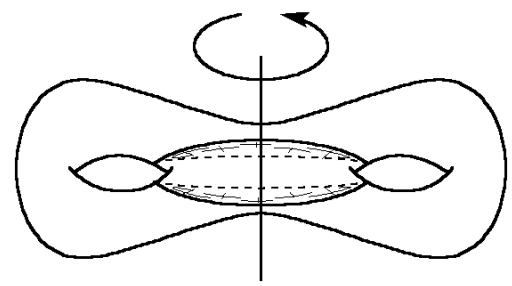

Figure C-4.

Hence, $G$ is a disk, and $G \cap \alpha$ consists of one point. Then:

Claim 2. $G$ is non-separating in $\tilde{T}$.

Proof. Assume that $G$ is separating. Then the closures of $\tilde{T}-G$ are solid tori $T_{1}, T_{2}$ with $\tau\left(T_{i}\right)=T_{i}$. Moreover the fixed point set of $\left.\tau\right|_{T_{i}}$ is an arc in $T_{i}$. But, by using $\mathbb{Z}_{2}$-equivariant loop theorem, it is easy to see that such $\left.\tau\right|_{T_{i}}$ does not exist.

By Claim 2, we see that we obtain a solid torus $T^{\prime}$ by cutting $\tilde{T}$ along $G$, and this shows that we obtain a 2 -string trivial tangle $(B, \beta)$, and obviously $D$ is $\beta$-incompressible.

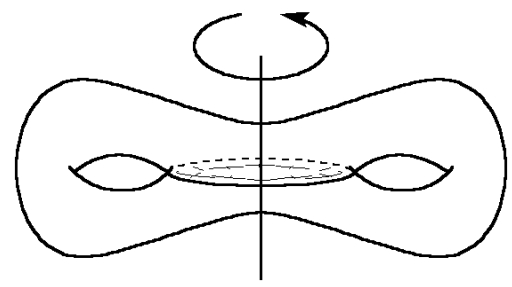

Figure C-5. 
Lemma C.3. Let $D_{1}, D_{2}$ be mutually disjoint non $\alpha$-parallel, $\alpha$-essential disks such that $D_{i} \cap \alpha(i=1,2)$ consists of two points. Then there exists an $\alpha$-compressing disk $D^{\prime}$ for $\partial T$ disjoint from $D_{1} \cup D_{2}$ such that $D^{\prime} \cap \alpha$ consists of one point. Moreover each $D_{i}$ is non-separating in $T$, and, by cutting $(T, \alpha)$ along $D^{\prime}$, we obtain 2 -string trivial tangle $(B, \beta)$, and $D_{1}, D_{2}$ are mutually non $\beta$-parallel, $\beta$-boundary parallel, $\beta$-incompressible disks in $(B, \beta)$.

Proof. Let $\tilde{D}_{i}$ be the lift of $D_{i}$ in $\tilde{T}(i=1,2)$. By Lemma A-1, we see that $\tilde{D}_{i}$ is an incompressible annulus in $\tilde{T}$. Let $\tilde{F}=c \ell\left(\partial \tilde{T}-N\left(\partial \tilde{D}_{1} \cup \partial \tilde{D}_{2}, \partial \tilde{T}\right)\right)$. The argument in the proof of Lemma C-2 works in this case to show that there is an equivariant compressing disk $G$ for $\tilde{F}$ such that $G$ is non-separating, and $G$ intersects $\tilde{\alpha}$ in one point. Then let $\tilde{T}^{\prime}$ be the solid torus obtained by cutting $\tilde{T}$ along $G$, and $G_{1}, G_{2}$ the copies of $G$ in $\partial \tilde{T}^{\prime}$. By Lemma B-0 (3), we see that there are annuli $A_{1}, A_{2}$ in $\partial \tilde{T}^{\prime}$ such that $A_{i}$ and $\tilde{D}_{i}$ are parallel $(i=1,2)$. Since $\tilde{D}_{1}, \tilde{D}_{2}$ are essential, we see that $G_{1} \subset A_{1}, G_{2} \subset A_{2}$. It is easy to see that this gives the conclusion of Lemma C-3.

Lemma C.4. Let $D$ be an $\alpha$-compressible disk such that $\partial D$ is $\alpha$-essential in $\partial T$, and $D \cap \alpha$ consists of two points. Then there is a disk $\Delta$ in $T$ such that $\Delta \cap D=\partial \Delta \cap D=\gamma$ an arc, and $\Delta \cap \alpha=c \ell(\partial \Delta-\gamma)$. Particularly, if $D$ is separating in $T$, then $D$ separates $(T, \alpha)$ into $\left(T^{\prime}, \alpha^{\prime}\right)$, and $\left(B^{\prime}, \alpha^{\prime \prime}\right)$ such that $\alpha^{\prime}$ is a trivial arc in a solid torus $T^{\prime}$. In this case, if $\left(B^{\prime}, \alpha^{\prime \prime}\right)$ happens to be a rational tangle, then $\left(B^{\prime}, \alpha^{\prime \prime}\right)$ is an $\alpha$-boundary parallelism.

Proof. Let $\tilde{D}$ be the lift of $D$ in $\tilde{T}$. By Lemma A-1, we see that $\tilde{D}$ is a compressible annulus in $\tilde{T}$. It is easy to see that there is a compressing disk $\tilde{\Delta}$ for $\tilde{D}$ such that $\tau(\tilde{\Delta})=\tilde{\Delta}$, hence $\tilde{\alpha} \subset \tilde{\Delta}$. Then the projection of $\tilde{\Delta}$ gives $\Delta$. Since $\partial D$ is $\beta$-essential, we see that, by compressing $\tilde{D}$ along $\tilde{\Delta}$, we obtain two meridian disks, say $D_{1}$ and $D_{2}$, which are mutually parallel in $\tilde{T}$. Suppose that $D$ is separating in $T$. Then $\tilde{D}$ is also separating in $\tilde{T}$, and the closures of the components of $\tilde{B}-\left(D_{1} \cup D_{2}\right)$ consist of $B^{3}$ and $T^{\prime}$, where $B^{3}$ is a 3 -ball, and $T^{\prime}$ is a solid torus. Then the closure of a component of $\tilde{T}-\tilde{D}$, say $H$, is obtained from $T^{\prime}$ by adding a 1-handle, and hence $H$ is a genus two handlebody. Now we consider the closure of the other component of $\tilde{T}-\tilde{D}$, say $E$. Then $E$ is obtained from $B^{3}$ by removing a regular neighborhood of an arc properly embedded in $B^{3}$, and taking the closure. This shows that $E$ is homeomorphic to the exterior of a knot in $S^{3}$ by a homeomorphism such that $E \cap \partial T$ is a regular neighborhood of a meridian loop of the knot in $\partial E$. Suppose that $E$ is a solid torus, i.e., $\left(B^{\prime}, \alpha^{\prime \prime}\right)$ is a rational tangle. Then the knot is a trivial knot. Since $E \cap \partial T$ is a regular neighborhood of a meridian loop of the knot, we see that $E$ is a $\partial$-parallelism for $\tilde{D}$, and by Lemma A-3, we see that $D$ is $\alpha$-boundary parallel. 


\section{References}

[1] J.M. Boardman, Singularities of differentiable maps, Publ. Math. I. H. E. S., 33 (1967), 21-57.

[2] F. Bonahon and J.-P. Otal, Scindements de Heegaard des espaces lenticulaires, Ann. Sci. Ec. Norm. Sup., 16(4) (1983), 451-466.

[3] G. Burde and H. Zieschang, Knots, Walter de Gruyter, Berlin, New York, 1985.

[4] A. Casson and C. Gordon, Reducing Heegaard splittings, Topology and its Applications, 27 (1987), 275-283.

[5] J. Cerf, Sur les difféomorphismes de la sphère de dimension trois $\left(\Gamma_{4}=0\right)$, Lecture Notes in Math., 53 (1968), Springer-Verlag, Berlin and New York.

[6] H. Doll, A generalized bridge number for links in 3-manifolds, Math. Ann., 294 (1992), 701-717.

[7] M. Golubitsky and V. Guillemin, Stable mappings and their singularities, Graduate Texts in Math., 14 (1973), Springer-Verlag, New York, Heidelberg, Berlin.

[8] W. Haken, Some results on surfaces in 3-manifolds, Studies in Modern Topology, Math. Assoc. Amer., (1968), 39-98.

[9] J. Hempel, 3-manifolds, Princeton University Press, Princeton, N. J., 1976.

[10] M.W. Hirsch, Differential topology, Graduate Texts in Math., 33 (1976), SpringerVerlag, New York, Heidelberg, Berlin.

[11] W. Jaco, Lectures on three-manifold topology, CBMS Regional Conf. Ser. in Math., 43 (1980).

[12] P.K. Kim and J.L. Tollefson, Splitting the P.L. involutions of nonprime 3-manifolds, Michigan Math. J., 27 (1980), 259-274.

[13] T. Kobayashi, Structures of the Haken manifolds with Heegaard splittings of genus two, Osaka J. Math., 21 (1984), 437-455.

[14] H. Levine, Classifying immersions into $\mathbf{R}^{4}$ over stable maps of 3-manifolds into $\mathbf{R}^{2}$, Lect. Notes in Math., 1157 (1985), Springer-Verlag, Berlin, Heidelberg, New York, Tokyo.

[15] J.N. Mather, Stability of $C^{\infty}$ mappings. V: Transversality, Adv. Math., 4 (1970), 301-336.

[16] J.W. Morgan and H. Bass (Eds.), The Smith Conjecture, Pure and Applied Math., 112 (1984), Academic Press.

[17] K. Morimoto and M. Sakuma, On unknotting tunnels for knots, Math. Ann., 289 (1991), 143-167.

[18] H. Rubinstein and M. Scharlemann, Comparing Heegaard splittings of non-Haken 3-manifolds, Topology, 35 (1996), 1005-1026.

[19] _ Comparing Heegaard splittings-the bounded case, preprint.

[20] H. Schubert, Knoten mit zwei Brücken, Math. Zeit., 66 (1956), 133-170.

[21] F. Waldhausen, Über Involutionen der 3-Sphäre, Topology, 8 (1969), 81-91. 
[22] H. Whitney, On singularities of mappings of Euclidean spaces. I. Mappings of the plane into the plane, Ann. of Math., 62 (1955), 374-410.

Received April 1, 1998 and revised June 24, 1999. The authors were supported by Grantin-Aid for Scientific Research, Ministry of Education, Science and Culture, Japan.

Department of Mathematics

NARA WOMEN'S UNIVERSITY

KITA-UOYA NiSHIMACHI

NARA 630

JAPAN

E-mail address: tsuyoshi@cc.nara-wu.ac.jp

Department of Mathematics, Faculty of Science

HiROSHIMA UNIVERSITY

HigASHI-HiRoshima 739

JAPAN

E-mail address: saeki@top2.math.sci.hiroshima-u.ac.jp 